NASA Technical Memorandum 106351

ICOMP-93-30

$1 N-64$

$1893 \% 3$

Analysis, Approximation, and Computation of a Coupled Solid/Fluid Temperature Control Problem

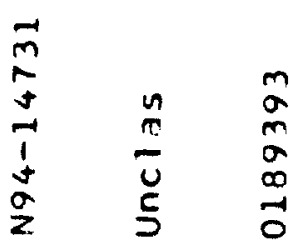

Max D. Gunzburger

Institute for Computational Mechanics in Propulsion

Lewis Research Center

Cleveland, Ohio

and Virginia Polytechnic Institute and State University

Blacksburg, Virginia

and

Hyung C. Lee

Virginia Polytechnic Instituie and State University

Blacksburg, Virginia

$\varangle$

$\ddot{0}$

$z \stackrel{u s}{\alpha}$

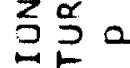

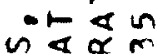

ニே山

ज马응

$>$ 皮

本要出向

रुण

은

$=2 \frac{3}{4}=$

जी.

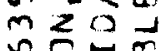

됭

두

T的告

$\sum \Sigma$

중ㅁㅁ

\&

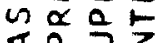

كaz

z웡

September 1993

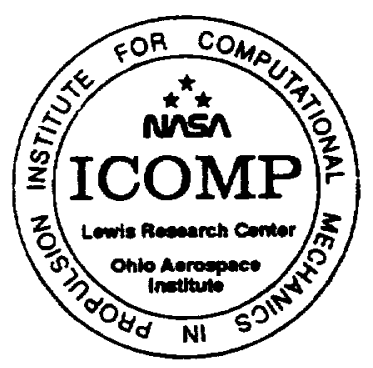




\title{
ANALYSIS, APPROXIMATION AND COMPUTATION OF A COUPLED \\ SOLID/FLUID TEMPERATURE CONTROL PROBLEM
}

\author{
Max D. Gunzburger \\ Institute for Computational Mechanics in Propulsion \\ Lewis Research Center \\ Cleveland, Ohio 44135 \\ and \\ Virginia Polytechnic Institute and State University \\ Blacksburg, Virginia 24061 \\ and \\ Hyung $C$. Lee \\ Virginia Polytechnic Institute and State University \\ Blacksburg, Virginia 24061
}

\begin{abstract}
An optimization problem is formulated motivated by the desire to remove temperature peaks, i.e., "hot spots", along the bounding surfaces of containers of fluid flows. The heat equation of the solid container is coupled to the energy equations for the fluid. Heat sources can be located in the solid body, the fluid, or both. Control is effected by adjustments to the temperature of the fluid at the inflow boundary. Both mathematical analyses and computational experiments are given.
\end{abstract}

\section{INTRODUCTION}

We suppose that the regular bounded domain $\Omega$ in $\mathbf{R}^{2}$ is made up of two subdomains $\Omega_{1}$ and $\Omega_{2}$ separated by an interface $\Gamma_{w}$, with the result that $\Omega=\Omega_{1} \cup \Omega_{2} \cup$ $\Gamma_{w}$ (see Figure 1). The solid material occupies a sub-domain $\Omega_{1}$ having a boundary $\Gamma_{1} \cup \Gamma_{2} \cup \Gamma_{3} \cup \Gamma_{w}$ and the fluid flow occupies a domain $\Omega_{2}$ having a boundary $\Gamma_{c} \cup \Gamma_{0} \cup \Gamma_{w} \cup \Gamma_{4}$. We have an inflow boundary $\Gamma_{c}$, an outflow boundary $\Gamma_{0}$, and a solid wall $\Gamma_{w}$. The geometry of all these boundary segments is prescribed, as are the inflow velocity $u_{c}$ and temperature $T_{c}$. At the outflow, one can impose one's favorite outflow boundary conditions. On the walls, we have the no slip boundary conditions for the velocity. Control is to be effected through heating and cooling along the boundary $\Gamma_{c}$.

The temperature is specified along the boundary $\Gamma_{c}$ and the heat-flux is specified along the boundary $\Gamma_{1} \cup \Gamma_{2} \cup \Gamma_{3} \cup \Gamma_{4} \cup \Gamma_{0}$. We assume that the flow is incompressible and convection driven so that buoyancy effects can be neglected, and thus temperature effects on the mechanical properties of the flow, i.e., the velocity and pressure, are negligible. We are interested in controls such that we get a desired temperature 


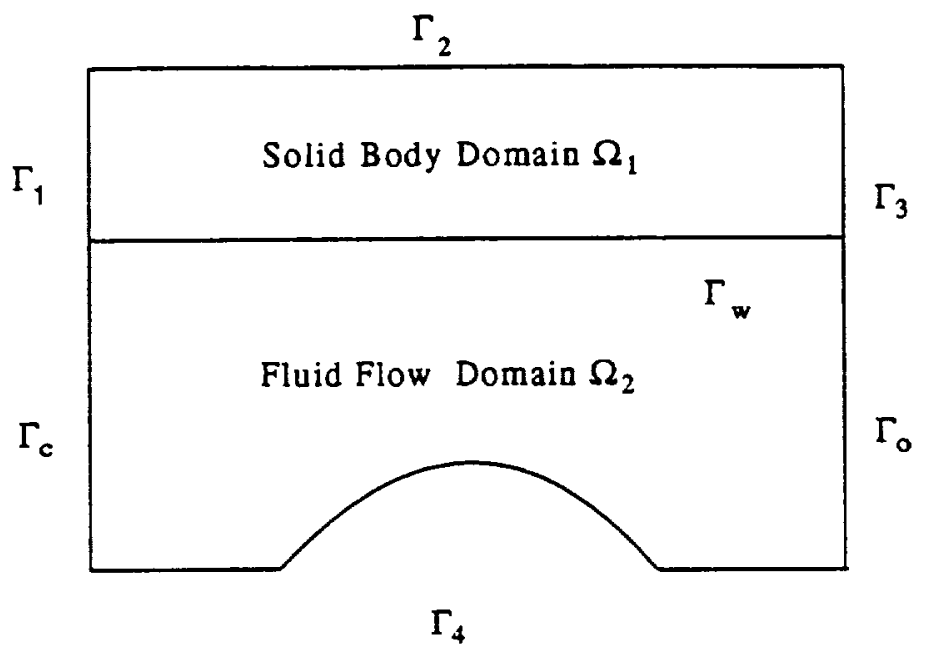

FIGURE 1. The Domain $\Omega$

along $\Gamma_{w}$ or a portion $\Gamma_{\sigma} \subset \Gamma_{w}$, and thus we assume that the flow is stationary. Other combinations of control and controlled surfaces are also possible.

As a result of our assumptions about the flow, the state variables, i.e., the velocity $u$, pressure $p$, temperature $T$, and control $g$ are required to satisfy the Navier-Stokes equations

$$
-\nu \Delta \mathbf{u}+(\mathbf{u} \cdot \nabla) \mathbf{u}+\nabla p=\mathbf{f} \text { in } \Omega_{\mathbf{2}}
$$

the incompressibility constraint

$$
\operatorname{div} \mathbf{u}=0 \text { in } \Omega_{2},
$$

and, for simplicity, the boundary condition

$$
\begin{aligned}
\mathbf{u} & =\mathbf{h} & & \text { on } \Gamma_{c}, \\
\mathbf{u} & =\mathbf{0} & & \text { on } \Gamma_{w} \cup \Gamma_{4}, \\
\frac{\partial \mathbf{u}}{\partial n} & =0 & & \text { on } \Gamma_{o},
\end{aligned}
$$

and the energy equations

$$
-\kappa_{1} \Delta T=\bar{Q}_{1} \quad \text { in } \Omega_{1}
$$

$$
-\kappa_{2} \Delta T+(\mathbf{u} \cdot \nabla) T=\bar{Q}_{2}+2 \mu\left(\nabla \mathbf{u}+\nabla \mathbf{u}^{T}\right):\left(\nabla \mathbf{u}+\nabla \mathbf{u}^{T}\right) \quad \text { in } \Omega_{2}
$$


with the boundary conditions

$$
\begin{aligned}
T & =g \quad \text { on } \Gamma_{c} \\
\frac{\partial T}{\partial n} & =0 \quad \text { on } \Gamma_{1} \cup \Gamma_{2} \cup \Gamma_{3} \cup \Gamma_{4} \cup \Gamma_{o} .
\end{aligned}
$$

The data functions $\mathbf{f}, \mathbf{h}, \bar{Q}_{1}$ and $\bar{Q}_{2}$ are assumed to be known. The constant $\nu$ is the kinematic viscosity coefficient of the fluid, and the constants $\kappa_{1}, \kappa_{2}$ and $\mu$ depend on the thermal conductivity coefficient, density, specific heat at constant volume, and viscosity coefficient of the fluid; see [17] for details.

Note that as a result of our assumptions about the flow, the mechanical equations (1.1)-(1.5) uncouple from the thermal equations (1.6)-(1.9). Indeed, (1.6)-(1.9) may be solved for $u$ and $p$ without regard of the temperature $T$. Thus, in the present context, the velocity field $u$, which is determined by solving (1.1)-(1.5), merely acts as a coefficient function and in the source term in (1.7).

We now define the optimal control problem to obtain our objective. For example, given a velocity field $u$, we would seek a temperature field $T$ and a control $g$ such that the functional

$$
\mathcal{J}(T, g)=\frac{1}{2 \gamma} \int_{\Gamma_{\sigma}}\left|T-T_{d}\right|^{2} d \Gamma+\frac{\kappa_{2} \delta}{2} \int_{\Gamma_{c}}\left(|g|^{2}+|\nabla, g|^{2}\right) d \Gamma
$$

is minimized subject to (1.6)-(1.9), where $\nabla$, denotes the surface gradient operator and $T_{d}$ is some desired temperature distribution, e.g., something close to the average temperature along $\Gamma_{\sigma}$ for the uncontrolled system. The non-negative parameters $\gamma$ and $\delta$ can be used to change the relative importance of the two terms appearing in the definition of $\mathcal{J}$ as well as to act as penalty parameter. Incidentally, the appearance of the control $g$ in the $\mathcal{J}$ is necessary because we are not imposing any a priori limits on the size of this control.

Under the realistic assumption that $\mathbf{u} \cdot \mathbf{n}=0$ on $\Gamma_{w} \cup \Gamma_{4}$ and $\mathbf{u} \cdot \mathbf{n} \geq 0$ on $\Gamma_{0}$, in this paper we prove the existence and uniqueness of optimal solutions and drive an optimality system, i.e., a set of equations from which the optimal control and state may be determined. Also, a finite element method is used to compute an approximate solution of the optimality system. We have also developed an iterative algorithm to compute the approximate solution.

We close this section by introducing some of the notation used in subsequent sections. Throughout, $C$ will denote a positive constant whose meaning and value changes with context. Also, $H^{s}(\mathcal{D}), s \in \mathbb{R}$, denotes the standard Sobolev space of order $s$ with respect to the set $\mathcal{D}$, where $\mathcal{D}$ is either the domain $\Omega$, or its boundary 
$\Gamma$, or part of that boundary. Of course, $H^{0}(\mathcal{D})=L^{2}(\mathcal{D})$. Dual spaces will be denoted by $(\cdot)^{*}$.

Of particular interest will be the space

$$
H^{1}(\Omega)=\left\{S \in L^{2}(\Omega): \frac{\partial S}{\partial x_{k}} \in L^{2}(\Omega) \quad \text { for } k=1,2\right\}
$$

and the subspace

$$
H_{D}^{1}(\Omega)=\left\{S \in H^{1}(\Omega): S=0 \text { on } \Gamma_{c}\right\}
$$

For functions defined on $\Gamma_{c}$, we will use the subspace

$$
W\left(\Gamma_{c}\right)=\left\{g \in H^{1}\left(\Gamma_{c}\right) \mid g=0 \text { at } \bar{\Gamma}_{c} \cap \bar{\Gamma}_{1}\right\} .
$$

Norms of functions belonging to $H^{s}(\Omega), H^{s}(\Gamma)$ and $H^{s}\left(\Gamma_{c}\right)$ are denoted by $\|\cdot\|_{s, \Omega}$, $\|\cdot\|_{s, \Gamma}$ and $\|\cdot\|_{s, \Gamma_{c}}$, respectively. Of particular interest are the $L^{2}(\Omega)$-norm $\|\cdot\|_{0, \Omega}$, the semi-norm

$$
|T|_{1, \Omega}^{2}=\sum_{j=1}^{2}\left\|\frac{\partial T}{\partial x_{j}}\right\|_{0, \Omega}^{2}
$$

and norm

$$
\|T\|_{1, \Omega}^{2}=|T|_{1, \Omega}^{2}+\|T\|_{0, \Omega}^{2}
$$

defined for functions belonging to $H^{1}(\Omega)$. Also, we are interested in the semi-norm $|\cdot| 1, r_{c}$, defined by

$$
|g|_{1, \Gamma_{c}}^{2}=\int_{\Gamma_{c}}\left|\nabla_{s} g\right|^{2} d \Gamma_{c}
$$

and norm

$$
\|g\|_{1, \Gamma_{c}}^{2}=|g|_{1, \Gamma_{c}}^{2}+\|g\|_{0, \Gamma_{c}}^{2}
$$

defined for functions belonging to $H^{1}\left(\Gamma_{c}\right)$ and $W\left(\Gamma_{c}\right)$.

We define, for $(T S) \in L^{1}(\Omega)$,

$$
(T, S)_{\Omega}=\int_{\Omega} T S d \Omega
$$

and, for $(p q) \in L^{1}(\Gamma)$,

$$
(p, q)_{\Gamma}=\int_{\Gamma} p q d \Gamma
$$

Thus, the inner product in $L^{2}(\Omega)$ is denoted by $(\cdot, \cdot)_{\Omega}$, that in $L^{2}(\Gamma)$ by $(\cdot, \cdot)_{\Gamma}$. The notation of (1.18)-(1.19) will also be employed to denote pairing between Sobolev spaces and their duals. 
We will use the bilinear forms, for $i=1,2$,

$$
a_{i}(T, S)=\kappa_{i} \int_{\Omega_{i}} \nabla T \cdot \nabla S d \Omega \quad \forall T, S \in H^{1}(\Omega)
$$

with the result that

$$
a(T, S)=\sum_{i=1}^{2} \kappa_{i} \int_{\Omega_{i}} \nabla T \cdot \nabla S d \Omega=a_{1}(T, S)+a_{2}(T, S),
$$

and the trilinear form

$$
c(\mathbf{u}, T, S)_{\Omega_{2}}=\int_{\Omega_{2}}(\mathbf{u} \cdot \nabla T) S d \Omega \quad \forall \mathbf{u} \in \mathbf{H}^{\mathbf{1}}\left(\Omega_{2}\right) \text { and } \forall T, S \in H^{1}\left(\Omega_{2}\right)
$$

These forms are continuous in the sense that there exist constants $c_{i}>0$ and $c_{c}>0$ such that, for $i=1,2$

$$
\left|a_{i}(T, S)\right| \leq c_{i}\|T\|_{1, \Omega_{i}}\|S\|_{1, \Omega_{i}} \quad \forall T, S \in H^{1}(\Omega)
$$

$$
\begin{array}{r}
\left|c(\mathrm{u}, T, S)_{\Omega_{2}}\right| \leq c_{c}\|\mathbf{u}\|_{1, \Omega_{2}}\|T\|_{1, \Omega_{2}}\|S\|_{1, \Omega_{2}} \\
\forall \mathrm{u} \in \mathrm{H}^{1}\left(\Omega_{2}\right) \text { and } \forall T, S \in H^{1}(\Omega),
\end{array}
$$

and thus

$$
\begin{aligned}
|a(T, S)| & \leq\left|a_{1}(T, S)\right|+\left|a_{2}(T, S)\right| \\
& \leq c_{1}\|T\|_{1, \Omega_{1}}\|S\|_{1, \Omega_{1}}+c_{2}\|T\|_{1, \Omega_{2}}\|S\|_{1, \Omega_{3}} \\
& \leq\left(c_{1}+c_{2}\right)\|T\|_{1, \Omega}\|S\|_{1, \Omega} \quad \forall T, S \in H^{1}(\Omega) .
\end{aligned}
$$

Moreover, we have the coercivity property, for $i=1,2$, there exist constants $C_{i}>0$ such that

$$
a_{i}(T, T) \geq C_{i}\|T\|_{1, \Omega_{i}}^{2} \quad \forall T \in H^{1}(\Omega)
$$

and thus

$$
a(T, T)=a_{1}(T, T)+a_{2}(T, T) \geq \sum_{i=1}^{2} C_{i}\|T\|_{1, \Omega_{i}}^{2} \geq \min \left(C_{1}, C_{2}\right)\|T\|_{1, \Omega}^{2}
$$




\section{The Optimization Problem and the Existence of Optimal Solutions}

We begin by giving a precise statement of the optimization problem we consider. We will assume the domain $\Omega$ is in $\mathbf{R}^{2}$ and consists of two subdomains $\Omega_{1}$ and $\Omega_{2}$ such that $\Omega=\Omega_{1} \cup \Omega_{2} \cup \Gamma_{w}$. Let $g \in W\left(\Gamma_{c}\right)$ denote the boundary control and let $T \in H^{1}(\Omega)$ denote the state, i.e., the temperature field. The state and control variables are constrained to satisfy the system eqrefeq:asst1-(1.9), which we recast into the following weak form: Find $(T, t) \in H^{1}(\Omega) \times H^{-1 / 2}\left(\Gamma_{c}\right)$ such that

$$
a(T, S)+c(\mathbf{u}, T, S)_{\Omega_{2}}-(t, S)_{\mathbf{r}_{e}}=(Q, S)_{\Omega} \quad \forall S \in H^{1}(\Omega)
$$

and

$$
(T, R)_{\Gamma_{\epsilon}}-(g, R)_{\Gamma_{\epsilon}}=0 \quad \forall R \in H^{-1 / 2}\left(\Gamma_{c}\right),
$$

where we have introduced the simplifying notation

$$
Q=\left\{\begin{array}{cc}
\bar{Q}_{1} & \text { in } \Omega_{1}, \\
\bar{Q}_{2}+2 \mu\left(\nabla \mathbf{u}+\nabla \mathbf{u}^{T}\right):\left(\nabla \mathbf{u}+\nabla \mathbf{u}^{T}\right) & \text { in } \Omega_{2}
\end{array}\right.
$$

One may show that, in the distributional sense,

$$
t=\left.\kappa_{2} \nabla T \cdot \mathbf{n}\right|_{\Gamma_{c}}
$$

In (2.1)-(2.2), we introduced the Lagrange multiplier $t$ to enforce the boundary condition. This will be very useful in the proof of error estimates for finite element approximations.

First, we show that for each possible control function $g$, there is a unique corresponding state function $(T, t)$.

Lemma 2.1. For every $g \in W\left(\Gamma_{c}\right)$, there exists a unique $(T, t) \in H^{1}(\Omega) \times H^{-1 / 2}\left(\Gamma_{c}\right)$ such that (2.1)-(2.2) are satisfied. Moreover, there exists a constant $C=C(\Omega)$ such that

$$
\|T\|_{1, \Omega}+\|t\|_{-1 / 2, \Gamma_{c}} \leq C\left(\|Q\|_{-1, \Omega}+\|g\|_{1, \Gamma_{c}}\right)
$$

Proof. For given $g \in W\left(\Gamma_{c}\right),(2.1)-(2.2)$ is equivalent to

$$
\begin{gathered}
a(T, S)+c(\mathbf{u}, T, S)_{\Omega_{2}}=(Q, S)_{\Omega} \quad \forall S \in H_{D}^{1}(\Omega), \\
T=g \quad \text { on } \Gamma_{c}
\end{gathered}
$$

and

$$
t=\left.\kappa_{2} \nabla T \cdot \mathbf{n}\right|_{\Gamma_{c}}
$$


By the virtue of the trace theorem, let $\hat{T}$ in $H^{1}(\Omega)$ satisfy $\hat{T}=g$ on $\Gamma_{c}$ and examine the following problem: Find $T$ in $H^{1}(\Omega)$ such that

$$
\begin{aligned}
& T-\hat{T} \in H_{D}^{1}(\Omega), \\
& a(T-\hat{T}, S)+c(\mathbf{u}, T-\hat{T}, S)_{\Omega_{2}} \\
& \quad=(Q, S)_{\Omega}-a(\hat{T}, S)-c(\mathbf{u}, \hat{T}, S)_{\Omega_{2}} \quad \forall S \in H_{D}^{1}(\Omega) .
\end{aligned}
$$

Let $\tilde{T}=T-\hat{T} \in H_{D}^{1}(\Omega)$. From the assumption of $\mathbf{u}, i . e ., \mathbf{u} \cdot \mathbf{n}=0$ on $\Gamma_{w} \cup \Gamma_{4}$ and $\mathbf{u} \cdot \mathbf{n} \leq 0$ on $\Gamma_{o}$, we have that

$$
\begin{aligned}
c(\mathbf{u}, \tilde{T}, \tilde{T})_{\Omega_{2}} & =\frac{1}{2} \int_{\partial \Omega_{2}}(\mathbf{u} \cdot \mathbf{n}) \tilde{T}^{2} d \Gamma \\
& =\frac{1}{2} \int_{\Gamma_{0}}(\mathbf{u} \cdot \mathbf{n}) \tilde{T}^{2} d \Gamma \geq 0
\end{aligned}
$$

Thus, we have

$$
a(\tilde{T}, \tilde{T})+c(\mathbf{u}, \tilde{T}, \tilde{T})_{\Omega_{2}} \geq \min \left(C_{1}, C_{2}\right)\|\tilde{T}\|_{1, \Omega}^{2}
$$

Therefore, by the Lax-Milgram theorem there is a unique $\tilde{T} \in H_{D}^{1}(\Omega)$, i.e., $T=$ $\tilde{T}+\hat{T} \in H^{1}(\Omega)$ and the estimate

$$
\|T\|_{1, \Omega} \leq C\left(\|Q\|_{-1, \Omega}+\|g\|_{1 / 2, \Gamma_{c}}\right) \leq C\left(\|Q\|_{-1, \Omega}+\|g\|_{1, \Gamma_{c}}\right)
$$

holds. From the trace theorem and the theory of partial differential equations (see [4]), we have

$$
\|t\|_{-1 / 2, \Gamma_{e}} \leq C\left(\|T\|_{1, \Omega_{2}}+\left\|Q_{2}\right\|_{-1, \Omega_{2}}\right) \leq C\left(\|T\|_{1, \Omega}+\|Q\|_{-1, \Omega}\right),
$$

where $Q_{2}=\bar{Q}_{2}+2 \mu\left(\nabla \mathbf{u}+\nabla \mathbf{u}^{T}\right):\left(\nabla \mathbf{u}+\nabla \mathbf{u}^{T}\right)$. Then, (2.4) follows from above two estimates.

The admissibility set $\mathcal{U}_{a d}$ is defined by

$$
\begin{aligned}
\mathcal{U}_{a d}=\left\{(T, g) \in H^{1}(\Omega)\right. & \times W\left(\Gamma_{\varepsilon}\right): \mathcal{J}(T, g)<\infty, \text { and there exists a } \\
& \left.t \in H^{-1 / 2}\left(\Gamma_{\epsilon}\right) \text { and }(2.1)-(2.2) \text { is satisfied }\right\}
\end{aligned}
$$

Then, $(\hat{T}, \hat{g}) \in \mathcal{U}_{a d}$ is called an optimal solution if there exists $\epsilon>0$ such that

$$
\mathcal{J}(\hat{T}, \hat{g}) \leq \mathcal{J}(T, g) \quad \forall(T, g) \in \mathcal{U}_{a d} \text { satisfying }\|T-\hat{T}\|_{1}+\|g-\hat{g}\|_{1, \Gamma_{e}} \leq \epsilon .
$$

We now show the existence and uniqueness of optimal solutions.

Theorem 2.2. There exists a unique optimal solution $(\hat{T}, \hat{g}) \in \mathcal{U}_{a d}$. 
Proof. We first claim that $\mathcal{U}_{a d}$ is not empty. Let $g \equiv 0$ and then let $(\tilde{T}, \tilde{g}) \in$ $H^{1}(\Omega) \times W\left(\Gamma_{c}\right)$ be a solution of $(2.1)-(2.2)$; note that with $g=0,(2.1)-(2.2)$ is equivalent to

$$
\begin{gathered}
a(\tilde{T}, S)+(\mathbf{u}, \tilde{T}, S)_{\Omega_{3}}=(Q, S)_{\Omega} \quad \forall S \in H_{D}^{1}(\Omega), \\
\tilde{T}=0 \quad \text { on } \Gamma_{\epsilon},
\end{gathered}
$$

and

$$
t=\left.\kappa_{2} \nabla T \cdot \mathbf{n}\right|_{\Gamma_{c}} .
$$

By Lemma 2.1, $(\tilde{T}, \tilde{t})$ exists and $(\tilde{T}, 0) \in \mathcal{U}_{a d}$. Now let $\left\{T^{(n)}, g^{(n)}\right\}$ be a sequence in $\mathcal{U}_{\text {ad }}$ such that

$$
\begin{gathered}
a\left(T^{(n)}, S\right)+\left(\mathbf{u}, T^{(n)}, S\right)_{\Omega_{3}}-\left(t^{(n)}, S\right)_{\Gamma_{c}}=(Q, S)_{\Omega} \quad \forall S \in H^{1}(\Omega), \\
\left(T^{(n)}, R\right)_{\Gamma_{c}}-\left(g^{(n)}, R\right)_{\Gamma_{c}}=0 \quad \forall R \in H^{-1 / 2}\left(\Gamma_{c}\right),
\end{gathered}
$$

and

$$
\lim _{n \rightarrow \infty} \mathcal{J}\left(T^{(n)}, g^{(n)}\right)=\inf _{(T, g) \in U_{a d}} \mathcal{J}(T, g)
$$

for some $t^{(n)} \in H^{-1 / 2}\left(\Gamma_{c}\right)$. Then, using (1.10) and (2.13), we have that $\left\{\left\|g^{(n)}\right\|_{1, \Gamma_{c}}\right\}$ is uniformly bounded which in turn yields that $\left\{\left\|T^{(n)}\right\|_{1}\right\}$ and $\left\{\left\|t^{(n)}\right\|_{-1 / 2, \Gamma_{c}}\right\}$ are uniformly bounded. We may then extract subsequences such that

$$
\begin{aligned}
g^{(n)} & -\hat{g} \text { in } W\left(\Gamma_{c}\right) \\
T^{(n)} & -\hat{T} \text { in } H^{1}(\Omega) \\
t^{(n)} & -\hat{t} \text { in } H^{-1 / 2}\left(\Gamma_{c}\right) \\
T^{(n)} & \rightarrow \hat{T} \text { in } L^{2}(\Omega) \\
\left.T^{(n)}\right|_{\Gamma_{\sigma}} & \left.\rightarrow \hat{T}\right|_{\Gamma_{\odot}} \text { in } L^{2}\left(\Gamma_{\sigma}\right)
\end{aligned}
$$

for some $(\hat{T}, \hat{g}) \in H^{1}(\Omega) \times W\left(\Gamma_{c}\right)$. The last two convergence results above follow from the compact imbeddings $H^{1}(\Omega) \subset L^{2}(\Omega)$ and $H^{1 / 2}\left(\Gamma_{\sigma}\right) \subset L^{2}\left(\Gamma_{\sigma}\right)$. We may then easily pass to the limit in $(2.16)-(2.17)$ to determine that $(\hat{T}, \hat{g}, \hat{t})$ satisfies (2.1)(2.2). Now, by the weak lower semicontinuity of $\mathcal{J}(\cdot, \cdot)$, we conclude that $(\hat{T}, \hat{g})$ is an optimal solution, i.e.,

$$
\mathcal{J}(\hat{T}, \hat{g})=\inf _{(T, g) \in \mathcal{U}_{a d}} \mathcal{J}(T, g) .
$$


Thus, we have shown that an optimal solution belonging to $\mathcal{U}_{a d}$ exists. Finally, the uniqueness of the optimal solution follows from the convexity of the functional and the linearity of the constraint equations.

Let

$$
L=-\sum_{i=1}^{2} \frac{\partial}{\partial x_{i}}\left(\sum_{j=1}^{2} a_{i j} \frac{\partial}{\partial x_{j}}\right)+\sum_{i=1}^{2} c_{i} \frac{\partial}{\partial x_{i}}
$$

be a differential operator of the second order in divergence form on an open set $\Omega$ of $\mathbb{R}^{2}$. We introduce the bilinear differential operator associated with $L$

$$
L(T, S)=\sum_{i, j=1}^{2} a_{i j} \frac{\partial T}{\partial x_{i}} \frac{\partial S}{\partial x_{j}}+\sum_{i=1}^{2} c_{i} \frac{\partial T}{\partial x_{i}} S .
$$

Now, setting

$$
\begin{aligned}
& a_{11}=a_{22}=\left\{\begin{array}{ll}
\kappa_{1} & \text { on } \Omega_{1}, \\
\kappa_{2} & \text { on } \Omega_{2},
\end{array},\right. \\
& a_{12}=a_{21}=0 \quad \text { on } \Omega, \\
& c_{i}= \begin{cases}0 & \text { on } \Omega_{1}, \\
u_{i} & \text { on } \Omega_{2},\end{cases}
\end{aligned}
$$

where $\mathbf{u}=\left(u_{1}, u_{2}\right)$, we have the following theorems.

Theorem 2.3. Let $T$ be the solution of (2.5)-(2.6) and let $T_{1}$ and $T_{2}$ be the restrictions of $T$ to $\Omega_{1}$ and $\Omega_{2}$, then $T_{1}$ and $T_{2}$ are solutions of a transmission problem

$$
\begin{gathered}
a_{1}\left(T_{1}, S_{1}\right)=\left(Q_{1}, S_{1}\right)_{\Omega_{1}} \quad \forall S_{1} \in H^{1}\left(\Omega_{1}\right) \\
a_{2}\left(T_{2}, S_{2}\right)+c\left(\mathbf{u}, T_{2}, S_{2}\right)=\left(Q_{2}, S_{2}\right)_{\Omega_{2}} \quad \forall S_{2} \in H_{D}^{1}\left(\Omega_{2}\right) \\
T_{2}=g \quad \text { on } \Gamma_{c} \\
T_{1}=T_{2} \quad \text { on } \Gamma_{w}, \\
\kappa_{1} \frac{\partial T_{1}}{\partial n}+\kappa_{2} \frac{\partial T_{2}}{\partial n}=0 \quad \text { on } \Gamma_{w} .
\end{gathered}
$$

Proof. For the proof, see [12]. 
Theorem 2.4. If we suppose the restrictions of $Q$ in (2.5)-(2.6) to $\Omega_{1}$ and $\Omega_{2}$ are $C^{\infty}\left(\Omega_{1} \cup \Gamma_{w}\right)$ and $C^{\infty}\left(\Omega_{2} \cup \Gamma_{w}\right)$, respectively, and $g \in C^{\infty}$, then every solution in $H^{1}(\Omega)$ of (2.5)-(2.6) has its restrictions $T_{1}, T_{2}$ to $\Omega_{1}, \Omega_{2}$ are $C^{\infty}\left(\Omega_{1} \cup \Gamma_{w}\right)$, $C^{\infty}\left(\Omega_{2} \cup \Gamma_{w}\right)$, respectively, and therefore in particular the solutions of the transmission problem (2.22)-(2.26).

Proof. For the proof, see ([11], Proposition 9, p592).

We define the space $\tilde{H}^{s}(\Omega)$ for $s \geq 1$ by

$$
\tilde{H}^{s}(\Omega)=\left\{T \in H^{1}(\Omega)\|\| T \|_{\bar{H} \cdot(\Omega)}<\infty\right\},
$$

where

$$
\|T\|_{\tilde{H}^{\prime}(\Omega)}^{2}=\|T\|_{H^{1}(\Omega)}^{2}+\sum_{i=1}^{2}\|T\|_{H^{\prime}\left(\Omega_{i}\right)}^{2} .
$$

Theorem 2.5. Let $T \in H^{1}(\Omega)$ be the solution of the problem (2.1)-(2.2). Then, we have

$$
\|T\|_{\tilde{H}^{2}(\Omega)}+\|t\|_{H^{1 / 2}\left(\Gamma_{w}\right)} \leq C\|Q\|_{H^{0}(\Omega)}
$$

Proof. For the proof, see ([5], Theorem 8.5.1).

\section{First-Order Necessary Conditions for the Optimal Solution and an Optimal System}

We now proceed to derive the first-order optimality conditions associated with problem (2.14). The optimal control problem (2.14) is equivalent to the following minimization problem: Find $g \in W\left(\Gamma_{\varepsilon}\right)$ such that $\mathcal{K}(g):=\mathcal{J}(T(g), g)$ is minimized where $T(g) \in H^{1}(\Omega)$ is defined as solution of (2.1)-(2.2). By studying the Gâteaux derivative of the functional $\mathcal{K}(g)$, we can obtain the first-order necessary conditions for the optimal solution $(\hat{T}, \hat{g})$ in a straightforward manner. Let $\hat{g}$ be a solution of the minimization problem $\min _{g \in W\left(\Gamma_{c}\right)} \mathcal{K}(g)$, then for every $z \in W\left(\Gamma_{c}\right)$ we have

$$
\forall \lambda \in \mathbb{R}, \quad \mathcal{K}(\hat{g}+\lambda z) \geq \mathcal{K}(\hat{g})
$$

due to the definition of $\hat{g}$. In particular, we have,

$$
\forall \lambda>0, \quad \frac{\mathcal{K}(\hat{g}+\lambda z)-\mathcal{K}(\hat{g})}{\lambda} \geq 0
$$

and,

$$
\forall \lambda<0, \quad \frac{\mathcal{K}(\hat{g}+\lambda z)-\mathcal{K}(\hat{g})}{\lambda} \leq 0
$$


which implies that the Gâteaux derivative of $\mathcal{K}(\hat{g})$

$$
\frac{d \mathcal{K}(\hat{g})}{d g}=\frac{d \mathcal{J}(T(\hat{g}), \hat{g})}{\hat{g}}=0 .
$$

Lemma 3.1. The mapping $g \rightarrow T(g)$, from $W\left(\Gamma_{c}\right)$ into $H^{1}(\Omega)$, has a Gâteaux derivative $(d T(g) / d g) \cdot z$ in every direction $z \in W\left(\Gamma_{e}\right)$. Furthermore, $(d T(g) / d g) \cdot z=$ $V(z)$ is the solution of

$$
\begin{gathered}
a(V, S)+c(\mathbf{u}, V, S)_{\Omega_{2}}-(\eta, S)=0 \quad \forall S \in H^{1}(\Omega), \\
(V, R)_{\Gamma_{c}}=(z, R)_{\Gamma_{c}} \quad \forall R \in H^{-1 / 2}\left(\Gamma_{c}\right) .
\end{gathered}
$$

Proof. It is immediate from the linearity of (2.1)-(2.2).

Now, we derive an optimality system from the first-order necessary condition (3.4). For each fixed $g$, the derivative $d \mathcal{K}(g) / d g \cdot z$ for every direction $z \in W\left(\Gamma_{c}\right)$ may be easily computed

$$
\begin{aligned}
\frac{d \mathcal{K}(g)}{d g} \cdot z & =\kappa_{2} \delta \int_{\Gamma_{c}}(\nabla g \cdot \nabla z+g z) d \Gamma+\frac{1}{\gamma} \int_{\Gamma_{\sigma}}\left(T-T_{d}\right) V d \Gamma \\
& =\kappa_{2} \delta(\nabla g, \nabla z)_{\Gamma_{c}}+\kappa_{2} \delta(g, z)_{\Gamma_{c}}+\frac{1}{\gamma}\left(T-T_{d}, V\right)_{\sigma} \quad \forall z \in W\left(\Gamma_{c}\right),
\end{aligned}
$$

where for each $z \in W\left(\Gamma_{c}\right), V \in H^{1}(\Omega)$ is the solution of (3.5)-(3.6).

Let $(T, t) \in H^{1}(\Omega) \times H^{-1 / 2}\left(\Gamma_{c}\right)$ be the solution of $(2.1)-(2.2)$ and let $(\Phi, \tau) \in$ $H^{1}(\Omega) \times H^{-1 / 2}\left(\Gamma_{c}\right)$ be defined as the solution of the adjoint problem

$$
\begin{gathered}
a(Z, \Phi)+c(\mathrm{u}, Z, \Phi)_{\Omega_{2}}+(\tau, Z)_{\Gamma_{c}}=\frac{1}{\gamma}\left(Z, T-T_{d}\right)_{\Gamma_{c}} \quad \forall Z \in H^{1}(\Omega), \\
(W, \Phi)_{\Gamma_{c}}=0 \quad \forall W \in H^{-1 / 2}\left(\Gamma_{c}\right) .
\end{gathered}
$$

Setting $S=\Phi$ in (3.5)-(3.6) and $Z=V$ in (3.8)-(3.9), we have that

$$
(\tau, z)_{\Gamma_{c}}=\frac{1}{\gamma}\left(V, T-T_{d}\right)_{\Gamma_{\sigma}} .
$$

Thus, from the necessary condition (3.4), we see that the optimal value of the control $g$ satisfies

$$
(\nabla g, \nabla z)_{\Gamma_{c}}+(g, z)_{\Gamma_{c}}=-\frac{1}{\kappa_{2} \delta}(\tau, z)_{\Gamma_{c}} \quad \forall z \in W\left(\Gamma_{c}\right)
$$

Collecting the above results, we obtain the optimal system 


$$
\begin{gathered}
a(T, S)+c(\mathbf{u}, T, S)_{\Omega_{2}}-(t, S)_{\Gamma_{c}}=(Q, S)_{\mathbf{\Omega}} \quad \forall S \in H^{1}(\Omega), \\
(T, R)_{\mathbf{r}_{c}}-(g, R)_{\Gamma_{c}}=0 \quad \forall R \in H^{-1 / 2}\left(\Gamma_{c}\right) \\
a(Z, \Phi)+c(\mathbf{u}, Z, \Phi)_{\Omega_{2}}+(\tau, Z)_{\mathbf{r}_{c}}=\frac{1}{\gamma}\left(Z, T-T_{d}\right)_{\Gamma_{c}} \quad \forall Z \in H^{1}(\Omega) \\
(W, \Phi)_{\Gamma_{c}}=0 \quad \forall W \in H^{-1 / 2}\left(\Gamma_{c}\right)
\end{gathered}
$$

and

$$
(\nabla g, \nabla z)_{\Gamma_{c}}+(g, z)_{\Gamma_{c}}=-\frac{1}{\delta \kappa_{2}}(\tau, z)_{\Gamma_{c}} \quad \forall z \in W\left(\Gamma_{c}\right)
$$

Integration by parts may be used to show that the system (3.12)-(3.16) constitutes a weak formulation of the boundary value problem

$$
-\kappa_{1} \Delta T=\bar{Q}_{1} \quad \text { in } \Omega_{1},
$$

$$
\begin{gathered}
-\kappa_{2} \Delta T+(\mathbf{u} \cdot \nabla) T=\bar{Q}_{2}+2 \mu\left(\nabla \mathbf{u}+\nabla \mathbf{u}^{T}\right):\left(\nabla \mathbf{u}+\nabla \mathbf{u}^{T}\right) \quad \text { in } \Omega_{2}, \\
T=g \quad \text { on } \Gamma_{c}, \\
-\kappa_{1} \Delta \Phi=0 \quad \text { in } \Omega_{1}, \\
-\kappa_{2} \Delta \Phi-(\mathbf{u} \cdot \nabla) \Phi=0 \quad \text { in } \Omega_{2}, \\
\Phi=0 \quad \text { on } \Gamma_{c}, \\
\frac{\partial \Phi}{\partial n}=\frac{1}{\kappa_{2} \gamma}\left(T-T_{d}\right) \quad \text { on } \Gamma_{\sigma}
\end{gathered}
$$

and

$$
-\Delta, g+g=\left.\frac{1}{\delta} \nabla \Phi \cdot \mathbf{n}\right|_{\Gamma_{c}} \quad \text { on } \Gamma_{c}
$$




\section{Finite Element Approximation and Error Estimates}

A finite element discretization of the optimality system (3.12)-(3.16) is defined as follows. One may choose families of finite dimensional subspaces $V_{1}^{h} \subset H^{1}\left(\Omega_{1}\right)$, $V_{2}^{h} \subset H^{1}\left(\Omega_{2}\right)$ such that $\left.V_{1}^{h}\right|_{\Gamma_{\varpi}}=\left.V_{2}^{h}\right|_{\Gamma_{\omega}}$. These families are parameterized by the parameter $h$ that tends to zero; commonly, this parameter is chosen to be some measure of the grid size in a subdivision of $\Omega$ into finite elements. Let $V^{h}=V_{1}^{h} \cup V_{2}^{h}$ and $O^{h}=\left.V^{h}\right|_{\Gamma_{c}}$, i.e., $O^{h}$ consists of the restriction, to the boundary $\Gamma_{c}$, of the functions, belonging to $V^{h}$. For all choices of conforming finite element spaces, we then have that $V^{h} \subset H^{1}(\Omega)$ and $O^{h} \subset H^{-1 / 2}\left(\Gamma_{c}\right)$. Next, let $N^{h}=V^{h} \mid \Gamma_{c}$. Again, for all choices of conforming finite element spaces $V^{h}$ we have that $N^{h} \subset H^{1}\left(\Gamma_{c}\right)$. Let $N_{0}^{h}=N^{h} \cap W\left(\Gamma_{e}\right)$. For the subspaces $V_{1}^{h}, V_{2}^{h}, O^{h}$ and $N_{0}^{h}$, we assume the approximation properties: there exist an integer $k$ and a constant $C$, independent of $h, T_{1}, T_{2}, t$ and $g$, such that

$$
\begin{array}{ll}
\inf _{T_{1}^{h} \in V_{1}^{h}}\left\|T_{1}-T_{1}^{h}\right\|_{1} \leq C h^{m}\left\|T_{1}\right\|_{m+1, \Omega_{1}} & \forall T_{1} \in H^{m+1}\left(\Omega_{1}\right), 1 \leq m \leq k, \\
\inf _{T_{2}^{h} \in V_{2}^{h}}\left\|T_{2}-T_{2}^{h}\right\|_{1} \leq C h^{m}\left\|T_{2}\right\|_{m+1, \Omega_{2}} \quad \forall T_{2} \in H^{m+1}\left(\Omega_{2}\right), 1 \leq m \leq k, \\
\inf _{t^{h} \in O^{h}}\left\|t-t^{h}\right\|-1 / 2, \Gamma_{c} \leq C h^{m}\|t\|_{m-1 / 2, \Gamma_{c}} \quad \forall t \in H^{m-1 / 2}\left(\Gamma_{c}\right), 1 \leq m \leq k
\end{array}
$$

and

$$
\begin{aligned}
\inf _{g^{h} \in N_{0}^{h}}\left\|g-g^{h}\right\|_{s, \Gamma_{c}} \leq C h^{m-s+1 / 2}\|g\|_{m+1 / 2, \Gamma_{c}} & \\
& \forall g \in W\left(\Gamma_{c}\right), 1 \leq m \leq k, 0 \leq s \leq 1 .
\end{aligned}
$$

A finite element algorithm for determining approximations of the solution of the optimality system (3.12)-(3.16) is as follows: seek $T^{h} \in V^{h}, t^{h} \in O^{h}, g^{h} \in N_{0}^{h}$, $\Phi^{h} \in V^{h}$ and $\tau^{h} \in O^{h}$ such that

$$
\begin{gathered}
a\left(T^{h}, S^{h}\right)+c\left(\mathbf{u}, T^{h}, S^{h}\right) \Omega_{\Omega_{2}}-\left(t^{h}, S^{h}\right)_{\Gamma_{c}}=\left(Q, S^{h}\right) \quad \forall S^{h} \in V^{h}, \\
\left(T^{h}, R^{h}\right)_{\Gamma_{c}}-\left(g^{h}, R^{h}\right)_{\Gamma_{c}}=0 \quad R^{h} \in O^{h}, \\
\kappa_{2} \delta\left(g^{h}, K^{h}\right)_{\Gamma_{c}}+\kappa_{2} \delta\left(\nabla, g^{h}, \nabla, K^{h}\right)_{\Gamma_{c}}=-\left(K^{h}, \tau^{h}\right)_{\Gamma_{c}} \quad \forall K^{h} \in N_{0}^{h}, \\
a\left(Z^{h}, \Phi^{h}\right)+c\left(\mathbf{u}, Z^{h}, \Phi^{h}\right)_{\Omega_{2}}+\left(Z^{h}, \tau^{h}\right)_{\Gamma_{c}}=\frac{1}{\gamma}\left(Z^{h}, T^{h}-T_{d}\right)_{\Gamma_{c}} \quad \forall Z^{h} \in V^{h}
\end{gathered}
$$


and

$$
\left(W^{h}, \Phi^{h}\right)_{\Gamma_{c}}=0 \quad \forall W^{h} \in O^{h}\left(\Gamma_{c}\right) .
$$

The major task in this section is to obtain error estimates for the finite element solutions. It turns out to be convenient to apply the Brezzi-Rappaz-Raviart theory, even though our problem is linear. We introduce some spaces and operators, and verify the requirements of that theory. In the following discussion, the constants $\kappa_{1}$, $\kappa_{2}$ and $\delta$ will be held fixed. Thus, the system (3.12)-(3.16) and (4.5)-(4.9) depend on the single parameter $\gamma$.

Let $X=H^{1}(\Omega) \times H^{-1 / 2}\left(\Gamma_{c}\right) \times W\left(\Gamma_{c}\right) \times H^{1}(\Omega) \times H^{-1 / 2}\left(\Gamma_{c}\right), Y=\left(H^{1}(\Omega)\right)^{*} \times$ $H^{-1 / 2}\left(\Gamma_{c}\right) \times\left(H^{1}(\Omega)\right)^{*}$ and $Z=L^{2}(\Omega) \times L^{2}\left(\Gamma_{c}\right) \times L^{2}\left(\Gamma_{\sigma}\right)$. Let the operator $\mathcal{B} \in$ $\mathcal{L}(Y, X)$ be defined as follows: $\mathcal{B}(\tilde{Q}, \tilde{\Theta}, \tilde{P})=(\tilde{T}, \tilde{t}, \tilde{g}, \tilde{\Phi}, \tilde{\tau})$ for $(\tilde{Q}, \tilde{\Theta}, \tilde{P}) \in Y$ and $(\tilde{T}, \tilde{t}, \tilde{g}, \tilde{\Phi}, \tilde{\tau}) \in X$ if and only if

$$
\begin{gathered}
a(\tilde{T}, S)+c(\mathbf{u}, \tilde{T}, S)_{\Omega_{2}}-(\tilde{t}, S)_{\Gamma_{c}}=(\bar{Q}, S)_{\Omega} \quad \forall S \in H^{1}(\Omega), \\
(\tilde{T}, R)_{\Gamma_{c}}-(\Theta, R)_{\Gamma_{c}}=0 \quad \forall R \in H^{-1 / 2}\left(\Gamma_{c}\right), \\
\kappa_{2} \delta(\tilde{g}, K)_{\Gamma_{c}}+\kappa_{2} \delta(\nabla, \tilde{g}, \nabla)_{\Gamma_{c}}=-(K, \tilde{\tau})_{\Gamma_{c}} \quad \forall K \in W\left(\Gamma_{c}\right), \\
a(Z, \tilde{\Phi})+c(\mathbf{u}, Z, \tilde{\Phi})_{\Omega_{2}}+(Z, \tilde{\tau})_{\Gamma_{c}}=(Z, \tilde{P})_{\Omega} \quad \forall Z \in H^{1}(\Omega)
\end{gathered}
$$

and

$$
(W, \tilde{\Phi})_{\Gamma_{c}}=0 \quad \forall W \in H^{-1 / 2}(\Omega)
$$

Note that this system is weakly coupled. First, one may separately solve the problems (4.10)-(4.11) for $\tilde{T}$ and $\tilde{t}$ and (4.13)-(4.14) for $\tilde{\Phi}$ and $\tilde{\tau}$; then, one may solve the Laplacian problem (4.12) for $\bar{g}$.

Analogously, the operator $\mathcal{B}^{h} \in \mathcal{L}(Y ; X)$ is defined as follows: $\mathcal{B}^{h}(\tilde{Q}, \tilde{\Theta}, \tilde{P})=$ $\left(\tilde{T}^{h}, \tilde{t}^{h}, \tilde{g}^{h}, \tilde{\Phi}^{h}, \tilde{\tau}^{h}\right)$ for $(\tilde{Q}, \tilde{\Theta}, \tilde{P}) \in Y$ and $\left(\tilde{T}^{h}, \tilde{t}^{h}, \tilde{g}^{h}, \tilde{\Phi}, \tilde{\tau}^{h}\right) \in X^{h}$ if and only if

$$
\begin{gathered}
a\left(\tilde{T}^{h}, S^{h}\right)+c\left(\mathbf{u}, \tilde{T}^{h}, S^{h}\right)_{\Omega_{2}}-\left(\tilde{t}^{h}, S^{h}\right)_{\Gamma_{c}}=\left(\tilde{Q}, S^{h}\right)_{\Omega} \quad \forall S^{h} \in V^{h}, \\
\left(\tilde{T}^{h}, R^{h}\right)_{\Gamma_{c}}-\left(\Theta, R^{h}\right)_{\Gamma_{c}}=0 \quad \forall R^{h} \in O^{h}, \\
\kappa_{2} \delta\left(\tilde{g}^{h}, K^{h}\right)_{\Gamma_{c}}+\kappa_{2} \delta\left(\nabla_{s} \tilde{g}^{h}, \nabla_{s} K^{h}\right)_{\Gamma_{c}}=-\left(K^{h}, \tilde{\tau}^{h}\right)_{\Gamma_{c}} \quad \forall K^{h} \in N_{0}^{h}, \\
a\left(Z^{h}, \tilde{\Phi}^{h}\right)+c\left(\mathbf{u}, Z^{h}, \tilde{\Phi}^{h}\right)_{\Omega_{2}}+\left(Z^{h}, \tilde{\tau}^{h}\right)_{\Gamma_{c}}=\left(Z^{h}, \tilde{P}^{h}\right)_{\Omega} \quad \forall Z^{h} \in V^{h}
\end{gathered}
$$


and

$$
\left(W^{h}, \bar{\Phi}^{h}\right)_{\Gamma_{c}}=0 \quad \forall W^{h} \in O^{h}
$$

The system (4.15)-(4.19) is weakly coupled in the same sense as the system (4.10)(4.14).

Theorem 4.1. The second order elliptic problem (4.10)-(4.14) has a unique solution belonging to $X$. Assume that (4.1)-(4.4) hold. Then the discrete second order elliptic problem (4.15)-(4.19) has a unique solution belonging to $X^{h}$. Let $(\tilde{T}, \tilde{t}, \tilde{g}, \tilde{\Phi}, \tilde{\tau})$ and $\left(\tilde{T}^{h}, \tilde{t}^{h}, \tilde{g}^{h}, \tilde{\Phi}^{h}, \tilde{\tau}^{h}\right)$ denote the solutions of (4.10)-(4.14) and (4.15)-(4.19), respectively. Then we have that

$$
\begin{aligned}
\left\|\tilde{T}-\tilde{T}^{h}\right\|_{1}+\left\|\tilde{t}-\tilde{t}^{h}\right\|_{-1 / 2, \Gamma_{c}}+\left\|\tilde{g}-\tilde{g}^{h}\right\|_{1, \Gamma_{c}}+\left\|\tilde{\Phi}-\tilde{\Phi}^{h}\right\|_{1} & \\
& +\left\|\tilde{\tau}-\tilde{\tau}^{h}\right\|_{-1 / 2, \Gamma_{c}} \rightarrow 0 \text { as } h \rightarrow 0
\end{aligned}
$$

In addition, if $(\tilde{T}, \tilde{t}, \tilde{g}, \tilde{\Phi}, \tilde{\tau}) \in \tilde{H}^{2}(\Omega) \times H^{1 / 2}\left(\Gamma_{c}\right) \times H^{1}\left(\Gamma_{c}\right) \times \tilde{H}^{2}(\Omega) \times H^{1 / 2}\left(\Gamma_{c}\right)$, then

$$
\begin{gathered}
\left\|\tilde{T}-\tilde{T}^{h}\right\|_{1}+\left\|\tilde{t}-\tilde{t}^{h}\right\|_{-1 / 2, \Gamma_{c}}+\left\|\tilde{g}-\tilde{g}^{h}\right\|_{1, \Gamma_{c}} \\
+\left\|\tilde{\Phi}-\tilde{\Phi}^{h}\right\|_{1}+\left\|\tilde{\tau}-\tilde{\tau}^{h}\right\|_{-1 / 2, \Gamma_{c}} \\
\leq C h\left(\|\tilde{T}\|_{\bar{H}^{2}(\Omega)}+\|\bar{\Phi}\|_{\bar{H}^{2}(\Omega)}\right) .
\end{gathered}
$$

Proof. First, it follows from Lemma 2.1 that the two second order elliptic problems (4.10)-(4.11) and (4.13)-(4.14) each have a unique solution $(\tilde{T}, \tilde{t})$ and $(\tilde{\Phi}, \tilde{\tau})$ belonging to $H^{1}(\Omega) \times H^{-1 / 2}\left(\Gamma_{c}\right)$, respectively. From the Babuška's theory, the discrete second order elliptic problems (4.15)-(4.16) and (4.18)-(4.19) each have a unique solution $\left(\tilde{T}^{h}, \tilde{t}^{h}\right)$ and $\left(\tilde{\Phi}^{h}, \tilde{\tau}^{h}\right)$ belonging to $V^{h} \times O^{h}$, respectively. Moreover, we have that

$$
\left\|\tilde{T}-\tilde{T}^{h}\right\|_{1}+\left\|\tilde{t}-\tilde{t}^{h}\right\|_{-1 / 2, \Gamma_{c}} \rightarrow 0
$$

and

$$
\left\|\tilde{\Phi}-\tilde{\Phi}^{h}\right\|_{1}+\left\|\tilde{\tau}-\tilde{\tau}^{h}\right\|_{-1 / 2, \Gamma_{c}} \rightarrow 0
$$

as $h \rightarrow 0$, and if in addition $(\tilde{T}, \tilde{t}) \in \tilde{H}^{2}(\Omega) \times H^{1 / 2}\left(\Gamma_{c}\right)$ and $(\tilde{\Phi}, \tilde{\tau}) \in \tilde{H}^{2}(\Omega) \times$ $H^{1 / 2}\left(\Gamma_{c}\right)$, we have that

$$
\begin{aligned}
& \left\|\tilde{T}-\tilde{T}^{h}\right\|_{1}+\left\|\tilde{t}-\tilde{t}^{h}\right\|_{-1 / 2, \Gamma_{c}} \leq C h\|\tilde{T}\|_{\tilde{H}^{2}(\Omega)}, \\
& \left\|\tilde{\Phi}-\tilde{\Phi}^{h}\right\|_{1}+\left\|\tilde{\tau}-\tilde{\tau}^{h}\right\|_{-1 / 2, \Gamma_{c}} \leq C h\|\tilde{\Phi}\|_{\tilde{H}^{2}(\Omega)}
\end{aligned}
$$


Note that the problem (4.12) is a well known equation. Thus, we have that the problems (4.12) and (4.17) both have unique solutions, that

$$
\|\tilde{g}\|_{2, \Gamma_{c}} \leq C\|\tilde{\tau}\|_{1 / 2, \Gamma_{e}} \leq C\|\tilde{\Phi}\|_{\dot{H}^{\jmath}(\Omega)},
$$

$$
\left\|\tilde{g}-\tilde{g}^{h}\right\|_{1, \Gamma_{c}} \leq C\left(\left\|\tilde{g}-\hat{g}^{h}\right\|_{1, \Gamma_{e}}+\left\|\tilde{\tau}-\tilde{\tau}^{h}\right\|_{-1 / 2, \Gamma_{e}}\right) \quad \forall \hat{g}^{h} \in N_{0}^{h} .
$$

Using (4.4), (4.22) and (4.23) we then have that

$$
\left\|\tilde{g}-\tilde{g}^{h}\right\|_{1, \Gamma_{c}} \rightarrow 0 \text { as } h \rightarrow 0,
$$

and using (4.4), (4.24) and (4.25), we conclude that

$$
\left\|\tilde{g}-\tilde{g}^{h}\right\|_{1, \Gamma_{c}} \leq C h\|\Phi\|_{\tilde{H}^{2}(\Omega)} .
$$

Then, (4.22), (4.23) and (4.28) yield (4.20), and (4.24), (4.25) and (4.29) yield (4.21).

Let $\Lambda$ denote a compact subset of $\mathbb{R}_{+}$. We define the operator $\mathcal{G}$ from $\Lambda \times$ $X$ to $Y$ as follows: $\mathcal{G}(\gamma,(T, t, g, \Phi, \tau))=(\tilde{Q}, \tilde{\Theta}, \tilde{P})$ for every $(\tilde{Q}, \tilde{\Theta}, \tilde{P}) \in Y$ and $(\gamma,(T, t, g, \Phi, \tau)) \in \Lambda \times X$ if and only if

$$
\begin{gathered}
(\bar{Q}, S)_{\Omega}=-\left(\bar{Q}_{1}, S\right)_{\Omega_{1}}-\left(Q_{2}, S\right)_{\Omega_{2}} \quad \forall S \in H^{1}(\Omega), \\
(\bar{\Theta}, R)_{\Gamma_{c}}=(-g, R)_{\Gamma_{c}} \quad \forall R \in H^{-1 / 2}\left(\Gamma_{c}\right)
\end{gathered}
$$

and

$$
(\tilde{P}, Z)_{\Omega}=-\frac{1}{\gamma}\left(Z, T-T_{d}\right)_{\Gamma_{\bullet}} \quad \forall Z \in H^{1}(\Omega),
$$

where $Q_{2}=\bar{Q}_{2}+2 \mu\left(\nabla \mathbf{u}+\nabla \mathbf{u}^{T}\right):\left(\nabla \mathbf{u}+\nabla \mathbf{u}^{T}\right)$. The operator $\mathcal{G}$ is obviously of class $C^{\infty}$. The derivative of $\mathcal{G}$ with respective to $(T, t, g, \Phi, \tau)$, which we denote by $\mathcal{G}_{X}(\gamma,(T, t, g, \Phi, \tau))$, can be defined as follows:

$$
\mathcal{G}_{X}(\gamma,(T, t, g, \Phi, \tau))=\left(0,-g,-\frac{1}{\gamma} T\right)
$$

for every $(\gamma,(T, t, g, \Phi, \tau)) \in \Lambda \times X$. Furthermore, $\mathcal{G}(\gamma,(T, t, g, \Phi, \tau)) \in \mathcal{L}(X, Z)$. Since $\Lambda$ is a compact interval in $\mathbb{R}_{+}$and the constant $\kappa_{2}$ is fixed, we see that $\mathcal{G}$ and it's first and second Fréchet derivatives and all locally bounded maps.

It is easily seen that the optimality system (3.12)-(3.16) is equivalent to

$$
(T, t, g, \Phi, \tau)+\mathcal{B G}(\gamma,(T, t, g, \Phi, \tau))=0
$$

and that the discrete optimality system (4.5)-(4.9) is equivalent to

$$
\left(T^{h}, t^{h}, g^{h}, \Phi^{h}, \tau^{h}\right)+\mathcal{B}^{h} \mathcal{G}\left(\gamma,\left(T^{h}, t^{h}, g^{h}, \Phi^{h}, \tau^{h}\right)=0 .\right.
$$


Now, having verified the hypotheses of the Brezzi-Rappaz-Raviart theory, we may use that theory to deduce the estimate

$$
\begin{gathered}
\left\|T-T^{h}\right\|_{1}+\left\|t-t^{h}\right\|_{-1 / 2, \Gamma_{c}}+\left\|g-g^{h}\right\|_{1, \Gamma_{c}} \\
+\left\|\Phi-\Phi^{h}\right\|_{1}+\left\|\tau-\tau^{h}\right\|_{-1 / 2, \Gamma_{c}} \\
\leq C h\left(\|T\|_{\tilde{H}^{2}(\Omega)}+\|\Phi\|_{\hat{H}^{2}(\Omega)}\right) .
\end{gathered}
$$

We also have, from the theorem 2.5 , applied to (3.12)-(3.16), the regularity estimates

$$
\begin{aligned}
&\|T\|_{\tilde{H}^{2}(\Omega)}+\|t\|_{1 / 2, \Gamma_{c}}+\|g\|_{1, \Gamma_{c}}+\|\Phi\|_{\tilde{H}^{2}(\Omega)}+\|\tau\|_{1 / 2, \Gamma_{c}} \\
& \leq C\left(\|Q\|_{0}+\left\|T_{d}\right\|_{1 / 2, \Gamma_{\sigma}}\right) .
\end{aligned}
$$

The combination of (4.36) and (4.37) results in the following error estimates.

Theorem 4.2. Let $(T, t, \Phi, \tau)$ be the solution of (3.12)-(3.16) and let $\left(T^{h}, t^{h}, \Phi^{h}, \tau^{h}\right)$ be the solution of (4.5)-(4.9). Assume that $T, \Phi \in \tilde{H}^{2}(\Omega)$; also assume that (4.1)(4.2) hold. Then,

$$
\begin{gathered}
\left\|T-T^{h}\right\|_{1}+\left\|t-t^{h}\right\|_{-1 / 2, \Gamma_{c}}+\left\|g-g^{h}\right\|_{1, \Gamma_{c}} \\
+\left\|\Phi-\Phi^{h}\right\|_{1}+\left\|\tau-\tau^{h}\right\|_{-1 / 2, \Gamma_{c}} \\
\leq C h\left(\|Q\|_{0}+\left\|T_{d}\right\|_{1 / 2, \Gamma_{o}}\right),
\end{gathered}
$$

where $C$ is independent of $h, T, \Phi$.

We note that higher oreder estimates are possible if $T$ is smooth in each subdomain $\Omega_{1}$ and $\Omega_{2}$.

\section{Numerical Algorithm}

Let us consider the gradient method for the following minimization problem: Find $g \in W\left(\Gamma_{c}\right)$ such that $\mathcal{K}(g):=\mathcal{J}(T(g), g)$ is minimized where $T(g) \in H^{1}(\Omega)$ is defined as solution of (2.1)-(2.2).

The classical Simple Gradient Algorithm proceeds as follows:

$$
\begin{array}{ll}
\text { Given } & g^{(0)} ; \\
\text { define } & g^{(n+1)}=g^{(n)}-\frac{1}{\kappa_{2} \delta} \frac{d \mathcal{K}\left(g^{(n)}\right)}{d g} \quad \text { recursively. }
\end{array}
$$

Recall from $\S 3$ that for each fixed $g$, the derivative $d \mathcal{K}(g) / d g \cdot z$ may be computed

$$
\frac{d \mathcal{K}(g)}{d g} \cdot z=\kappa_{2} \delta\left(-\Delta_{s} g+g, z\right)+\frac{1}{\gamma}\left(T-T_{d}, V\right)_{\Gamma_{0}} \quad \forall z \in W\left(\Gamma_{c}\right),
$$

where for each $z \in W\left(\Gamma_{c}\right), V \in H^{1}(\Omega)$ is the solution of

$$
a(V, S)+c(\mathbf{u}, V, S)_{\Omega_{2}}=0 \quad \forall S \in H_{D}^{1}(\Omega),
$$




$$
V=z \text { on } \Gamma_{c} .
$$

From (3.13), we see that

$$
\left(-\kappa_{2} \frac{\partial \Phi}{\partial n}, z\right)_{\Gamma_{c}}=\frac{1}{\gamma}\left(V, T-T_{d}\right)_{\Gamma_{r}} .
$$

Thus, (5.1) may be replaced by

$$
\text { for } \quad \begin{aligned}
n=0,1,2, \ldots, \\
\text { set } \quad \begin{aligned}
g^{(n+1)} & =g^{(n)}-\frac{1}{\kappa_{2} \delta}\left(\kappa_{2} \delta\left(-\Delta_{s} g^{(n)}+g^{(n)}\right)-\left.\kappa_{2} \frac{\partial \Phi^{(n)}}{\partial n}\right|_{\Gamma_{c}}\right) \\
& =\Delta_{s} g^{(n)}+\left.\frac{1}{\delta} \frac{\partial \Phi^{(n)}}{\partial n}\right|_{\Gamma_{c}}
\end{aligned}
\end{aligned}
$$

where $\Phi^{(n)}$ is determined from $g^{(n)}$ through the relations

$$
\begin{gathered}
a\left(T^{(n)}, S\right)+c\left(\mathbf{u}, T^{(n)}, S\right)=(Q, S) \quad \forall S \in H_{D}^{1}(\Omega), \\
T^{(\mathfrak{n})}=g^{(n)} \text { on } \Gamma_{c},
\end{gathered}
$$

and

$$
\begin{aligned}
a\left(Z, \Phi^{(n)}\right)+c\left(\mathbf{u}, Z, \Phi^{(n)}\right) & =\frac{1}{\gamma}\left(Z, T^{(n)}-T_{d}\right)_{\Gamma_{\sigma}} \quad \forall Z \in H_{D}^{1}(\Omega), \\
\Phi^{(n)} & =0 \text { on } \Gamma_{e} .
\end{aligned}
$$

Therefore, we have the gradient algorithm results in the following iteration:

Choose $g^{(1)}$;

$$
\begin{gathered}
\text { for } n=1,2,3, \ldots, \quad \text { solve for } T^{(n)} \text { and } \Phi^{(n)} \text { from } \\
a\left(T^{(n)}, S\right)+c\left(\mathrm{u}, T^{(n)}, S\right)=(Q, S) \quad \forall S \in H_{D}^{1}(\Omega), \\
T^{(n)}=g^{(n)} \text { on } \Gamma_{c},
\end{gathered}
$$

and

$$
\begin{gathered}
a\left(Z, \Phi^{(n)}\right)+c\left(\mathbf{u}, Z, \Phi^{(n)}\right)=\frac{1}{\gamma}\left(Z, T^{(n)}-T_{d}\right)_{\Gamma_{\sigma}} \quad \forall Z \in H_{D}^{1}(\Omega), \\
\Phi^{(n)}=0 \quad \text { on } \Gamma_{c},
\end{gathered}
$$

then solve for $g^{(n+1)}$ from

$$
g^{(n+1)}=\Delta_{s} g^{(n)}+\left.\frac{1}{\delta} \frac{\partial \Phi^{(n)}}{\partial n}\right|_{\Gamma_{c}} .
$$

The convergence of the algorithm (5.11) is a direct consequence of the following lemma. 
Lemma 5.1. Let $\mathcal{K}$ be a real-valued functional on a Hilbert space $X$ with norm $\|\cdot\|_{X}$ and scalar product $(\cdot, \cdot)_{X}$. Suppose that there exist two constants $m$ and $M$ such that

i) $\mathcal{K}$ has a local minimum at a point $\bar{x}$ is of class $C^{2}$ in an open ball $B$ centered at $\bar{x}$,

ii) $\forall u \in B, \quad \forall(x, y) \in X \times X, \quad K^{\prime \prime}(u) \cdot(x, y) \leq M\|x\|_{X}\|y\|_{X}$,

iii) $\forall u \in B, \quad \forall x \in X, \quad \mathcal{K}^{\prime \prime}(u) \cdot(x, x) \geq m\|x\|_{X}^{2}$.

Let $\mathbf{R}$ denote the Riesz map, i.e. $\langle f, x\rangle=(\mathbf{R} f, x)_{X}$ for all $x \in X$ and all $f \in X^{*}$. Choose $x^{(0)} \in B$ and choose a sequence $\left\{\rho_{n}\right\}$ such that $0<\rho_{*} \leq \rho_{n} \leq \rho^{*}<2 m / M^{2}$. Then, the sequence $\left\{x^{(n)}\right\}$ defined by

$$
x^{(n)}=x^{(n-1)}-\rho_{n} \operatorname{RK} \mathcal{K}^{\prime}\left(x^{(n-1)}\right) \quad \text { for } \quad n=1,2, \ldots,
$$

converges to $\bar{x}$. Furthermore, if $B=X$ and $\bar{x}$ is a global minimum, then the gradient algorithm converges to $\bar{x}$ for any initial value $x^{(0)}$.

Proof. see, e.g., $[8]$

Theorem 5.2. Let $\left(T^{(n)}, \Phi^{(n)}, g^{(n)}\right)$ be the solution of $(5.11)$ and $(T, \Phi, g)$ the solution of (3.12)-(3.16). Then, if $\gamma \delta$ is sufficiently large, $g^{(n)} \rightarrow g$ and thus, $T^{(n)} \rightarrow T$ in $H^{1}(\Omega)$ and $\Phi^{(n)} \rightarrow \Phi$ in $H_{D}^{1}(\Omega)$ as $n \rightarrow \infty$.

Proof. In (5.11), we have the fixed parameter $\rho=1 /\left(\kappa_{2} \delta\right)$. For each $g \in W\left(\Gamma_{c}\right)$, the second Fréchet-derivative $\mathcal{K}^{\prime \prime}(g) \cdot(z, w)$ may be computed by

$$
\mathcal{K}^{\prime \prime}(g) \cdot(z, w)=\kappa_{2} \delta(\nabla w, \nabla z) \Gamma_{\Gamma_{c}}+\kappa_{2} \delta(w, z)_{\Gamma_{c}}+\frac{1}{\gamma}(U, V)_{\Gamma_{\sigma}}
$$

where $U \in H^{1}(\Omega)$ and $V \in H^{1}(\Omega)$ are the solution of

$$
\begin{gathered}
a(U, S)+c(\mathbf{u}, U, S)=0 \quad \forall S \in H_{D}^{1}(\Omega), \\
U=w \quad \text { on } \Gamma_{c},
\end{gathered}
$$

and of

$$
\begin{gathered}
a(V, S)+c(\mathbf{u}, V, S)=0 \quad \forall S \in H_{D}^{1}(\Omega), \\
U=z \quad \text { on } \Gamma_{c} .
\end{gathered}
$$


One can easily have that $\|U\|_{1} \leq C\|w\|_{1, \Gamma_{c}}$ and $\|V\|_{1} \leq C\|z\|_{1, \Gamma_{c}}$, where the value of the constant $C$ depends only on $\Omega$. Then,

$$
\begin{aligned}
\mathcal{K}^{\prime \prime}(g) \cdot(z, w) & \leq \kappa_{2} \delta\|w\|_{1, \Gamma_{c}}\|z\|_{1, \Gamma_{c}}+\frac{C}{\gamma}\|w\|_{1, \Gamma_{c}}\|z\|_{1, \Gamma_{c}} \\
& =\left(\kappa_{2} \delta+\frac{C}{\gamma}\right)\|w\|_{1, \Gamma_{c}}\|z\|_{1, \Gamma_{c}}
\end{aligned}
$$

and

$$
\begin{aligned}
\mathcal{K}^{\prime \prime}(g) \cdot(z, z) & =\kappa_{2} \delta\|z\|_{1, \Gamma_{c}}^{2}+\frac{1}{\gamma} \int_{\Gamma_{\bullet}}|V|^{2} d \Gamma \\
& \geq \kappa_{2} \delta\|z\|_{1, \Gamma_{c} \cdot}^{2}
\end{aligned}
$$

Setting $M=\kappa_{2} \delta+C / \gamma$ and $m=\kappa_{2} \delta$, we have, if $\gamma \delta>C /\left((\sqrt{2}-1) \kappa_{2}\right), 2 m / M^{2}>$ $\rho=1 /\left(\kappa_{2} \delta\right)$. The other hypotheses of Lemma 5.1 are easily shown to be valid. Hence, from that lemma, we obtain that

$$
g^{(n)} \rightarrow g \quad \text { in } W\left(\Gamma_{c}\right) \text { as } n \rightarrow \infty
$$

The desired convergence results follow from the a priori estimate (2.4).

Of course, the gradient algorithm (5.11) is applied to the discrete equations. Then, we have two contribution to the errors in the computational solution, the discretization error $T-T^{h}$ and the iteration error $T^{h}-T^{h(n)}$. In a practical point of view, it is difficult to calculate $\Delta_{s} g^{(n)}$ in the last equation of (5.11). By using (3.24), we can substitute (5.11) by the following iteration:

Choose $g^{(1)}$ and $\Phi^{(0)}$;

$$
\begin{gathered}
\text { for } n=1,2,3, \ldots, \quad \text { solve for } T^{(n)} \text { and } \Phi^{(n)} \text { from } \\
\begin{array}{c}
a\left(T^{(n)}, S\right)+c\left(u, T^{(n)}, S\right)=(Q, S) \quad \forall S \in H_{D}^{1}(\Omega), \\
T^{(n)}=g^{(n)} \text { on } \Gamma_{c},
\end{array}
\end{gathered}
$$

and

$$
\begin{gathered}
a\left(Z, \Phi^{(n)}\right)+c\left(\mathbf{u}, Z, \Phi^{(n)}\right)=\frac{1}{\gamma}\left(Z, T^{(n)}-T_{d}\right)_{\Gamma} \quad \forall Z \in H_{D}^{1}(\Omega), \\
\Phi^{(n)}=0 \quad \text { on } \Gamma_{\epsilon},
\end{gathered}
$$

then solve for $g^{(n+1)}$ from

$$
g^{(n+1)}=g^{(n)}-\frac{1}{\delta}\left(\left.\frac{\partial \Phi^{(n-1)}}{\partial n}\right|_{\Gamma_{c}}-\left.\frac{\partial \Phi^{(n)}}{\partial n}\right|_{\Gamma_{c}}\right)
$$




\section{NUMERICAL EXPERIMENTS}

Test 1 : We consider that the domain $\Omega$ is the unit square $(0,1) \times(0,1) \subset \mathbb{R}^{2}$, sub-domain $\Omega_{1}=(0,1) \times(0.75,1)$ and sub-domain $\Omega_{2}=(0,1) \times(0,0.75)$. Let $\Gamma_{\sigma}=(0.075,1) \times\{0.75\} \subset \Gamma_{w}=(0,1) \times\{0.75\}$ and $\Gamma_{c}=\{0\} \times(0,0.75)$ (see Figure 1 without the bump on the bottom boundary).

The finite element spaces $V_{1}^{h}$ and $V_{2}^{h}$ are chosen to be piecewise quadratic elements on a triangle mesh such that $V_{1}^{h}=V_{2}^{h}$ on $\Gamma_{w}$. We use the mesh size $h=1 / 12$ for all computation. Of course, calculations with varying mesh sizes were performed. In this paper, not being interested in the convergence history with varying mesh sizes, we do not report them.

Now, we consider the following problem

$$
\begin{gathered}
-\Delta T=6.0 \quad \text { on } \quad \Omega_{1}, \\
-2 \Delta T+(\mathbf{u} \cdot \nabla) T=0 \quad \text { on } \Omega_{2}, \\
T=1+g \quad \text { on } \Gamma_{c}, \\
\frac{\partial T}{\partial n}=0 \quad \text { on } \quad \partial \Omega \backslash \Gamma_{c},
\end{gathered}
$$

where the velocity $u$ is the solution of the Navier-Stokes equations

$$
-\Delta \mathbf{u}+(\mathbf{u} \cdot \nabla) \mathbf{u}+\nabla p=\mathbf{0} \text { in } \Omega_{2}
$$

the incompressibility constraint

$$
\operatorname{div} u=0 \text { in } \Omega_{2},
$$

and the boundary condition

$$
\begin{aligned}
\mathbf{u}=\mathrm{h} & \text { on } \Gamma_{c}, \\
\mathbf{u}=0 & \text { on } \Gamma_{w} \cup \Gamma_{4}, \\
\frac{\partial u_{1}}{\partial n}=0 & \text { and } \quad u_{2}=0 \text { on } \Gamma_{o},
\end{aligned}
$$

where $\mathbf{u}=\left(u_{1}, u_{2}\right)$ and $\mathbf{h}=\left(1.5 y-2 y^{2}, 0\right)$. To get approximate solutions for the Navier-Stokes solutions, we use the Taylor-Hood finite element on the domain $\Omega_{2}$. Actually, we have simple solutions $\mathbf{u}=\left(1.5 y-2 y^{2}, 0\right)$ of the above Navier-Stokes problems. 


\begin{tabular}{|c|c|c|c|}
\hline & $\|T-1.2\|_{0, \Gamma_{s}}$ & $\|g\|_{\Gamma_{c}}$ & $\mathcal{J}(T, g)$ \\
\hline$\gamma=1, \delta=1$ & $2.88669 \times 10^{-2}$ & 15.63322 & 15.64765 \\
$\gamma=0.01, \delta=0.003$ & $1.93423 \times 10^{-3}$ & 78.80300 & 0.333120 \\
\hline
\end{tabular}

TABLE 1. The numerical results for Test 1.

Note that since all data in (6.1)-(6.9) are sufficiently smooth in each domain $\Omega_{i}$ for $i=1,2$, we may assume that $T \in \tilde{H}^{s}(\Omega), s \geq 3$ from the theorem 2.4 . Thus we may use piecewise quadratic elements for the finite element approximation for the fast convergence with respect to $h$.

When $g=0$ in (6.3), we say that the problem (6.1)-(6.9) is an uncontrolled problem. The numerical solution of the uncontrolled problem is shown in Figure 2 and Figure 3 in which one can see that the temperature is above 2.0 on $(0.3,1) \times$ $\{0.75\}$ and even higher in the domain $(0.3,1) \times(0.75,1)$.

Now, we try to get the desired temperature distribution along $\Gamma_{\sigma}$. One can choose any reasonable desired temperature $T_{d}$ on $\Gamma_{\sigma}$, but we choose the parameter $T_{d}=1.2$ on $\Gamma_{\sigma}$, thus we have

$$
\mathcal{J}(T, g)=\frac{1}{2 \gamma} \int_{\Gamma_{\sigma}}|T-1.2|^{2} d \Gamma+\delta \int_{\Gamma_{c}}\left(|g|^{2}+|\nabla s g|^{2}\right) d \Gamma
$$

For the various choices of the parameters $\gamma$ and $\delta$ appearing in the functional (6.10), the computations were performed. In this paper, we report the numerical results for the cases

(1) $\gamma=\delta=1$,

(2) $\gamma=0.01$ and $\delta=0.003$.

The costs are shown in the Table 6 .

In Figure 4-7, we plot the surfaces of the temperature $T$ and adjoint state $\Phi$ for each case. If one chooses the relatively small $\gamma \delta$, then one can have the relatively small value of $\|T-1.2\|_{0, \Gamma_{\bullet}}$.

Further reinforcement of our conclusions can be obtained from Figure 8 and 9 in which are found contour plots of the temperature $T$ and adjoint state $\Phi$.

In Figure 10, we plot the approximate optimal control $g^{h}$ on the boundary $\Gamma_{c}$. In Figure 11, we compare the temperature distribution on $\Gamma_{\sigma}$ in the uncontrolled case with the optimal temperature distributions in the controlled cases.

Test 2 : We solve the problem $(6.1)-(6.9)$ with $\mathbf{h}=\left(1.5 y-2 y^{2}, 0\right)$ on the domain $\Omega$ which has a bumped boundary (see Figure 1 and 12). We assume that all parameters 


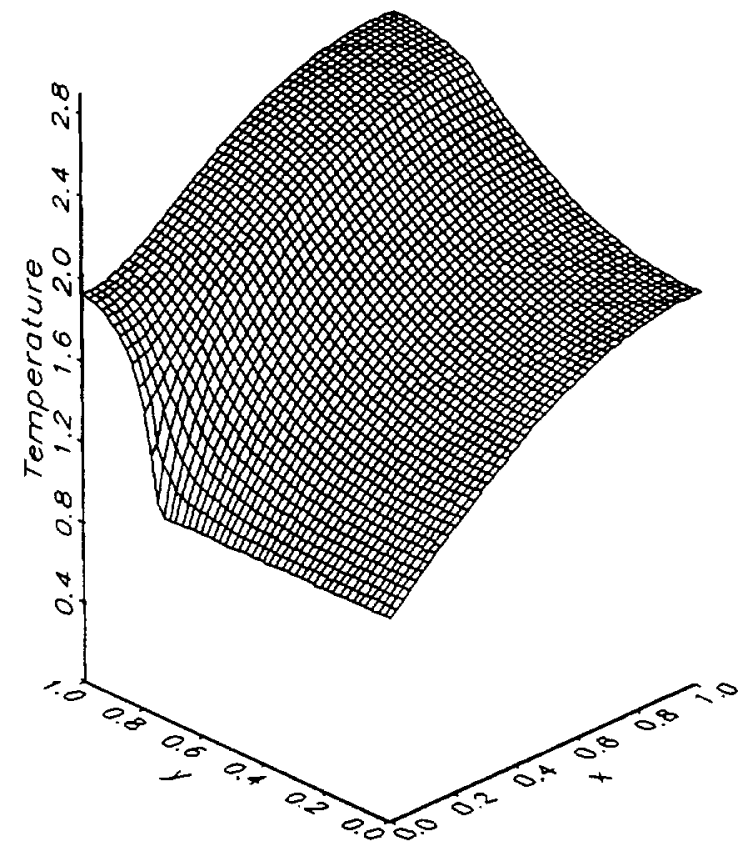

FIGURE 2. The temperature surface plot for the uncontrolled problem.

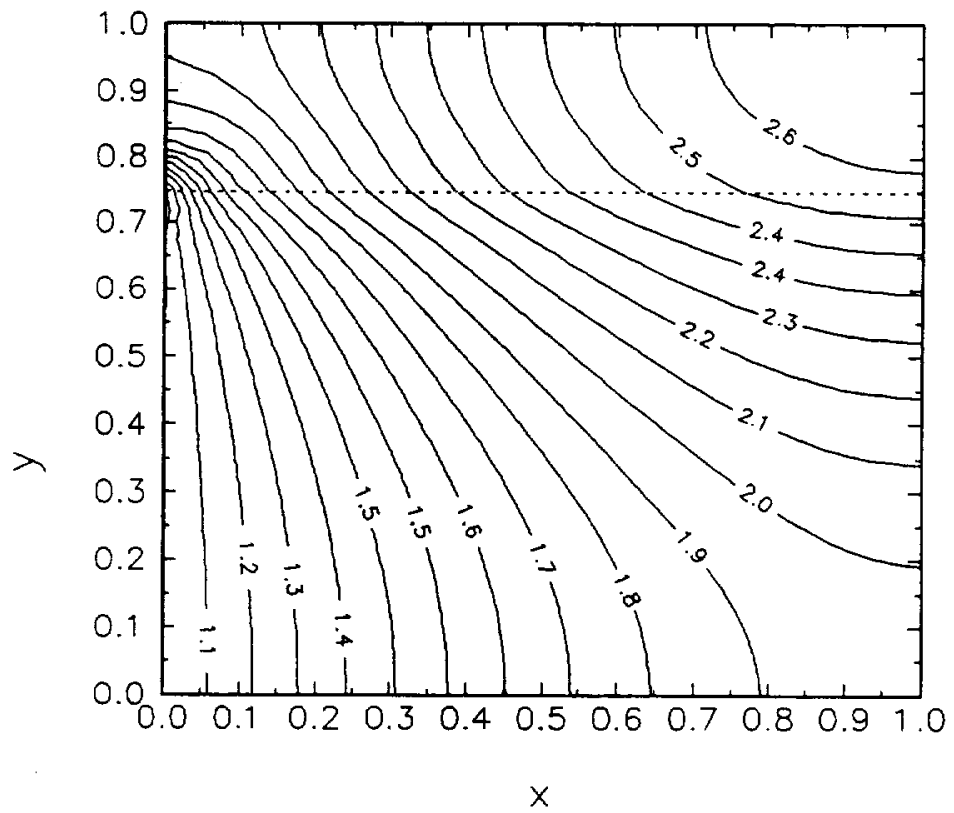

FIGURE 3. The temperature contour plot for the uncontrolled problem. 


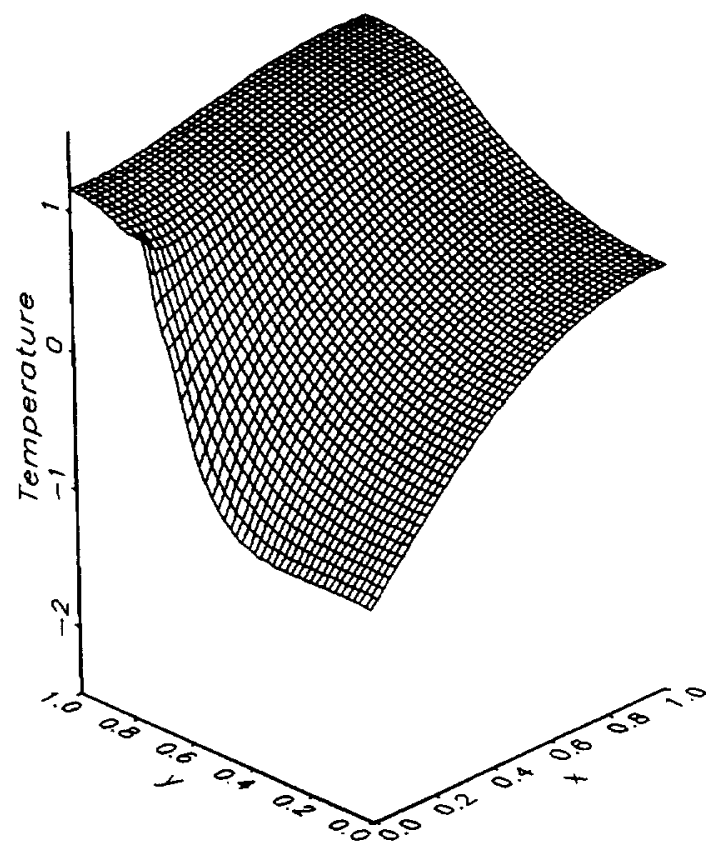

FigurE 4. The surface plot for the temperature $T(\gamma=\delta=1)$.

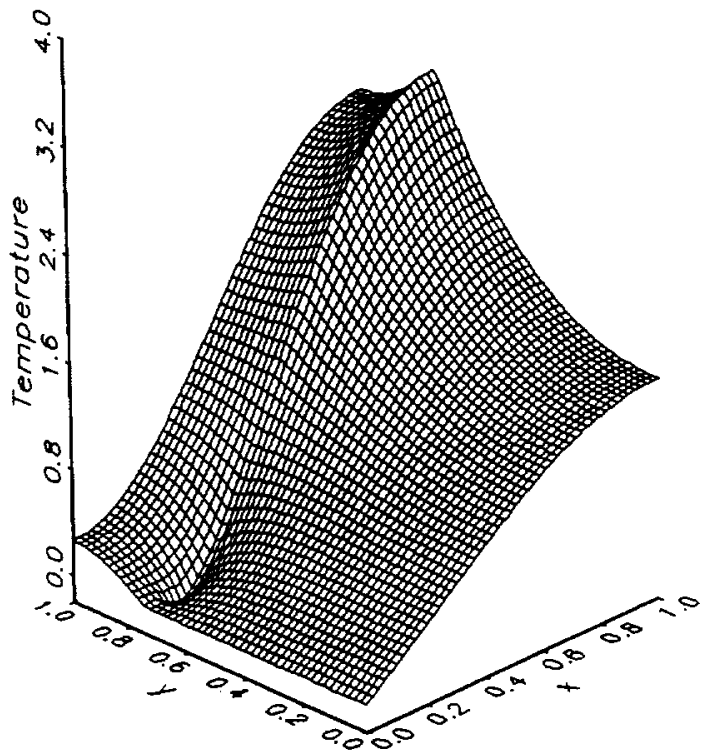

FIgURE 5. The surface plot for the adjoint state $\Phi(\gamma=\delta=1)$. 


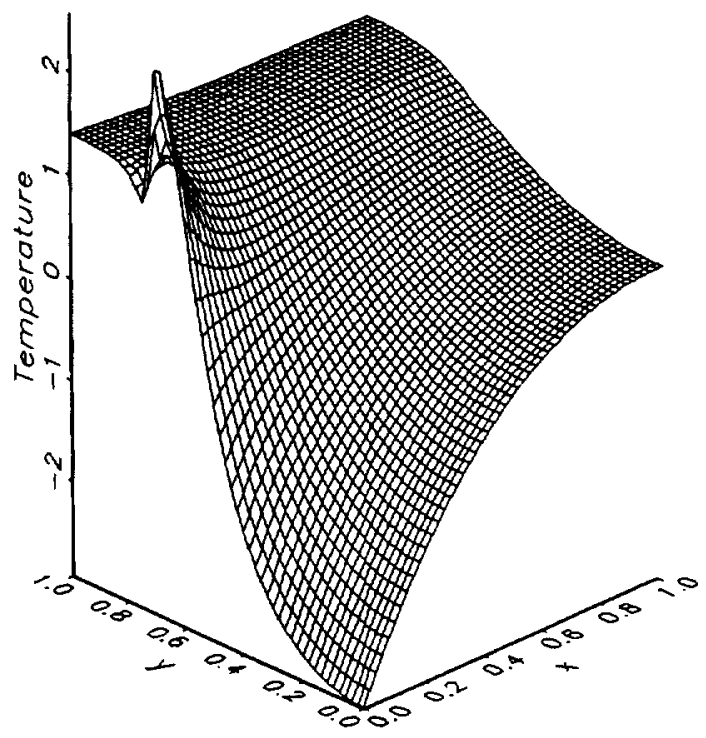

FIGURE 6. The surface plot for the temperature $T(\gamma=0.01, \delta=0.003)$.

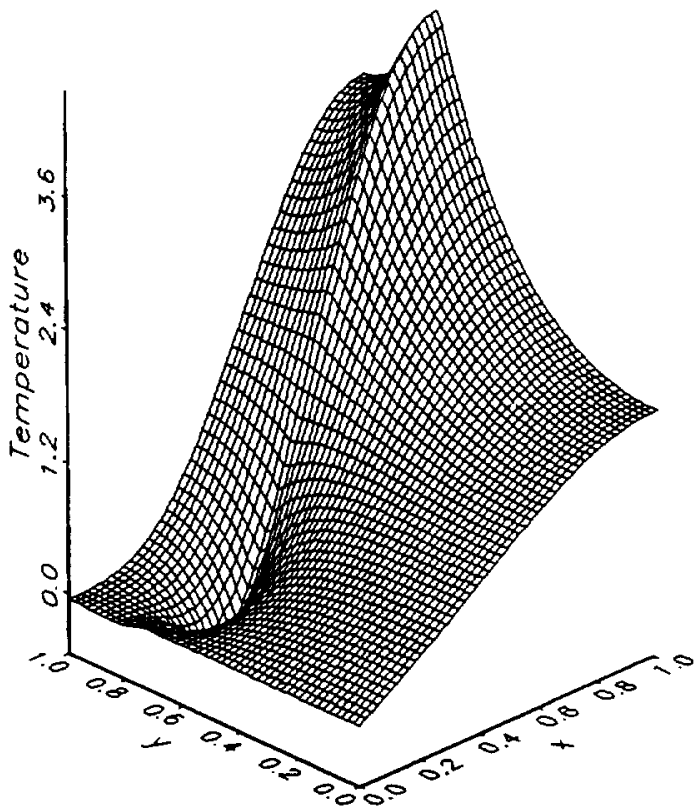

FIGURE 7. The surface plot for the adjoint state $\Phi(\gamma=0.01, \delta=0.003)$. 

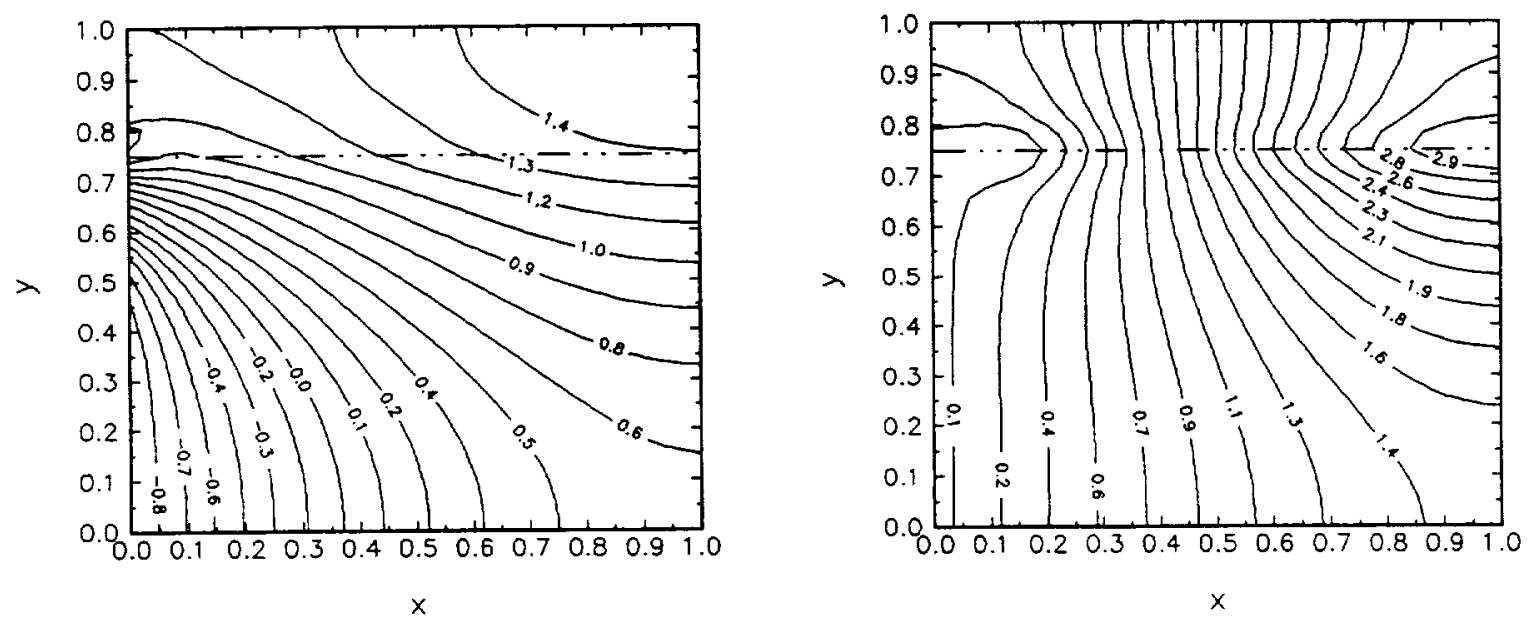

FIG URE 8. The contour plots for the temperature $T$ (left) and adjoint state $\Phi$ (right) $(\gamma=\delta=1)$.
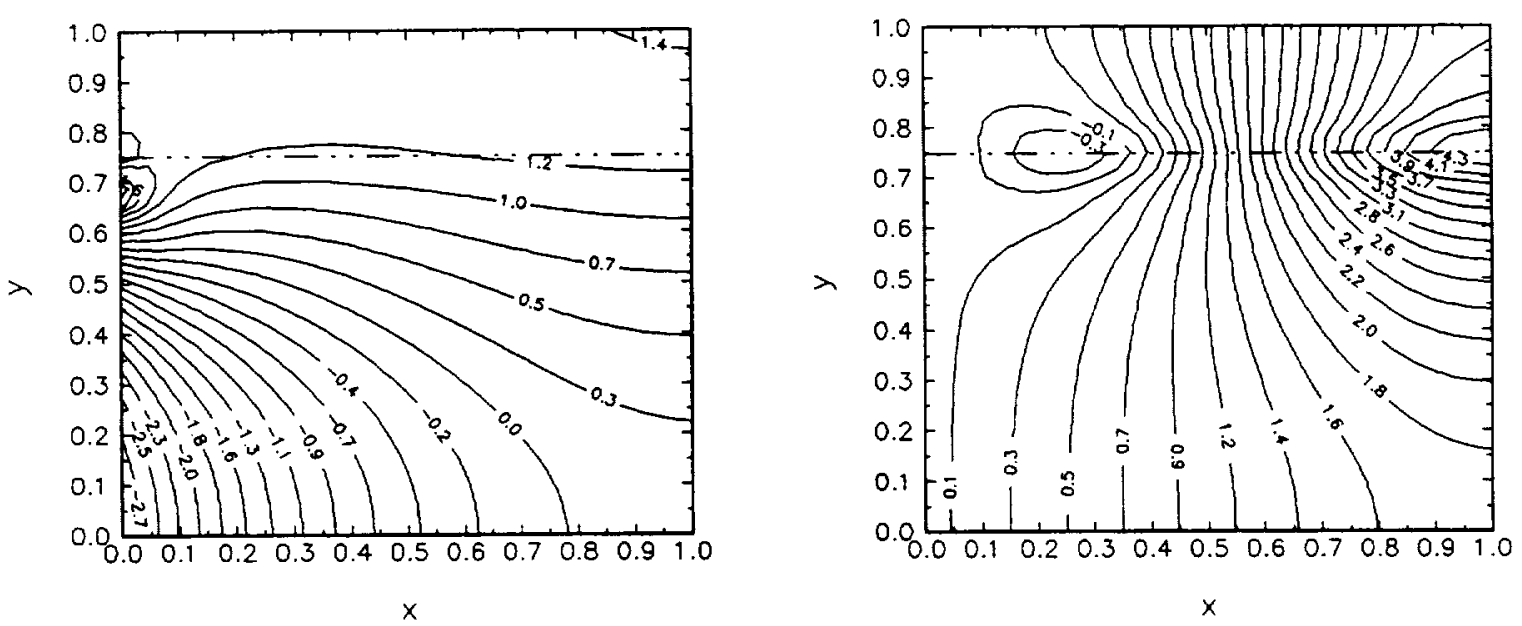

FIGURE 9. The contour plots for the temperature $T$ (left) and adjoint state $\Phi$ (right) $(\gamma=0.01, \delta=0.003)$. 


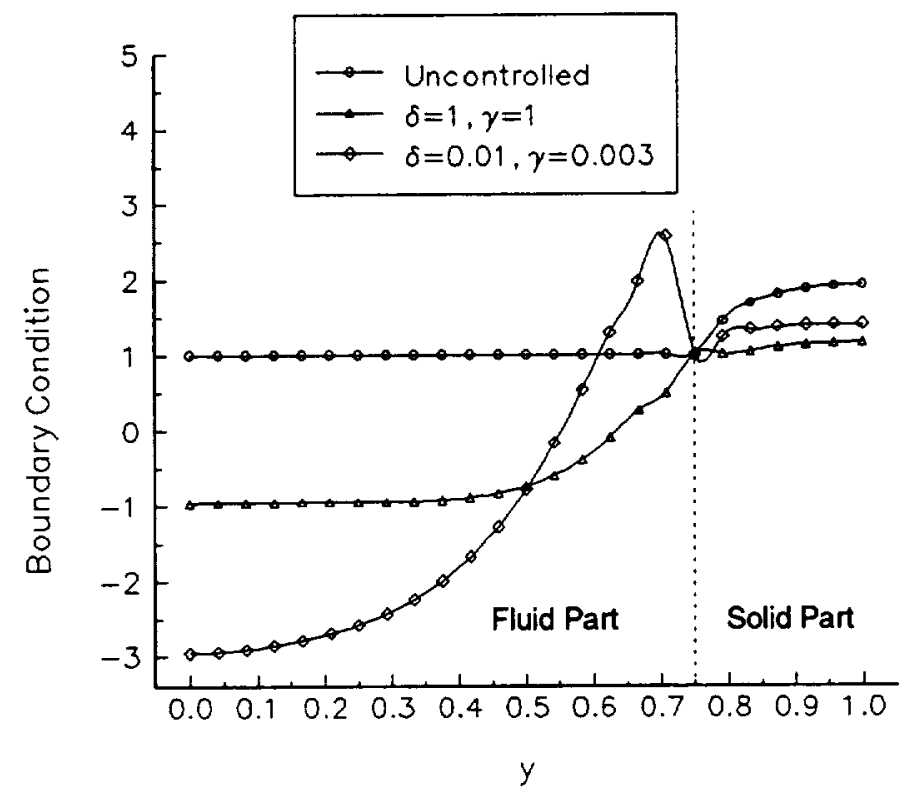

FIGURE 10. The optimal controls on $\Gamma_{c}$.

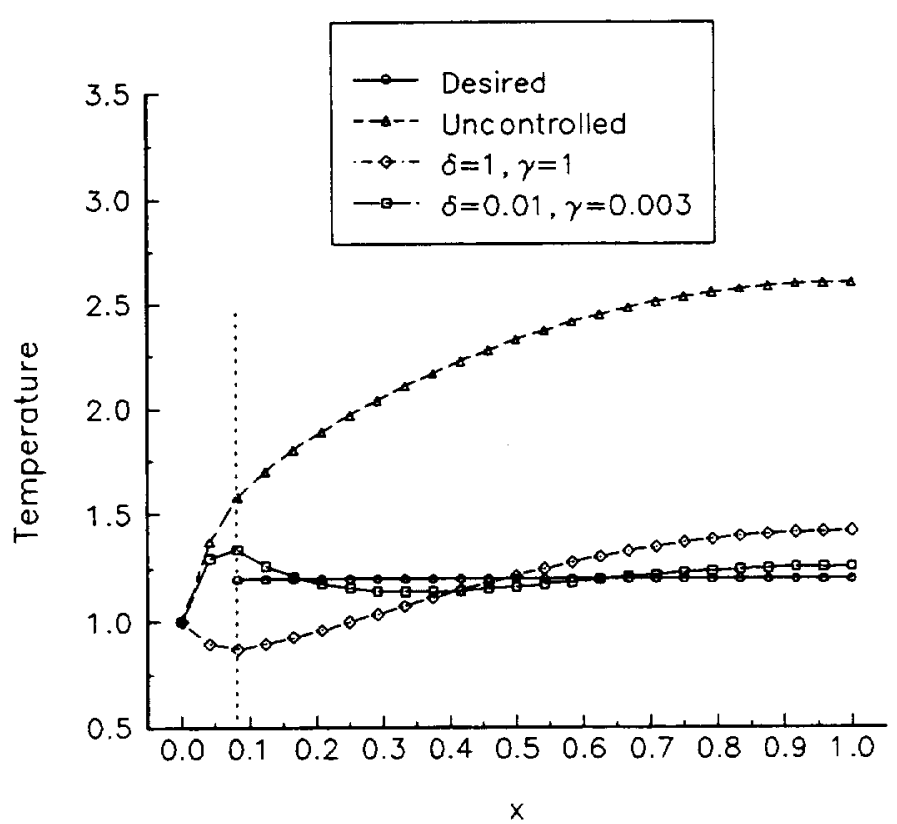

FIGURE 11. The temperature distributions on $\Gamma_{w}$. 


\begin{tabular}{|c|c|c|c|}
\hline & $\|\bar{T}-1.2\|_{0, \Gamma_{\sigma}}$ & $\|g\|_{\Gamma_{c}}$ & $\mathcal{J}(T, g)$ \\
\hline$\gamma=1, \delta=1$ & $3.77877 \times 10^{-2}$ & 18.65837 & 18.67727 \\
$\gamma=0.01, \delta=0.002$ & $3.13342 \times 10^{-3}$ & 117.14224 & 0.390956 \\
\hline
\end{tabular}

TABLE 2. The numerical results for Test 2.

and data are the same as in Test 1 . To get the approximate solutions for the NavierStokes equations, we also use the Taylor-Hood finite element on the domain $\Omega_{2}$.

We report the numerical results for the cases

(1) $\gamma=\delta=1$,

(2) $\gamma=0.01$ and $\delta=0.002$.

The costs are shown in the Table 6 . We get the almost same results as in Test 1 except that we need a little more control $g$ on $\Gamma_{c}$. Thus, even though the fluid flow is moderately complicated, given any $\epsilon>0$, we can have $\gamma$ and $\delta$ such that $\left\|T-T_{d}\right\|_{0, \Gamma_{\sigma}}<\epsilon$ when $\gamma \delta$ is sufficiently small.

In Figure 12, we plot the temperature contour for the uncontrolled problem. In Figure 13-14, we have the contour plots of the temperature $T$ and adjoint state $\Phi$ for each cases. Finally, Figure 15-16 display the approximate optimal control $g^{h}$ along $\Gamma_{c}$ and the temperature distributions on $\Gamma_{o}$, respectively.

Remarks : For the case $\gamma=\delta=1$, it was found that $10-15$ iterations were sufficient to get the optimal control $g$. Since $\nu=1$ and maximum velocity is 1 , the control $g$ affects the temperature distribution on $\Gamma_{\sigma}$ very weakly. For the case that $\gamma \delta$ is small, for example $\gamma \delta<0.1$, our gradient method does not converge. Thus, we need to adjust the iteration step size. In such case, we need a significant number of iterations. Thus, one may look for an efficient iteration algorithm. But the good news is that the iteration algorithm requires only one $\mathrm{LU}$ factorization and the same number of back and forward substitution as the iteration number , i.e., a comparable number of floating point operations relative to that required for solving the full coupled system (4.5)-(4.9). Of course we assume that $h$ is sufficiently small. 


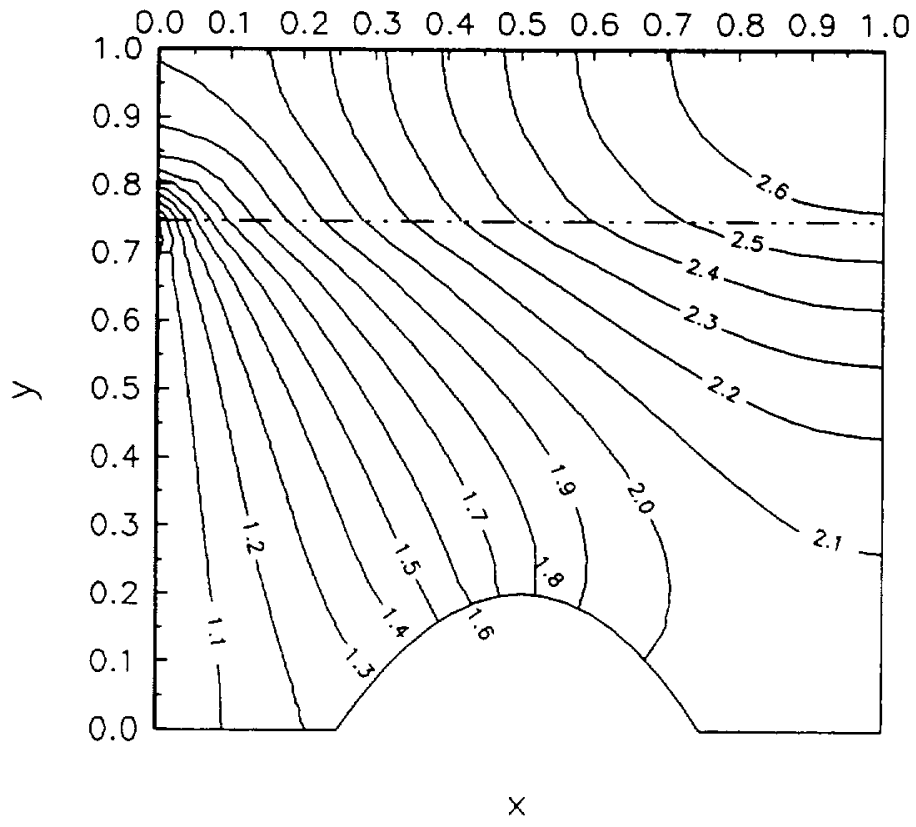

FIGURE 12. The temperature contour plot for the uncontrolled problem.
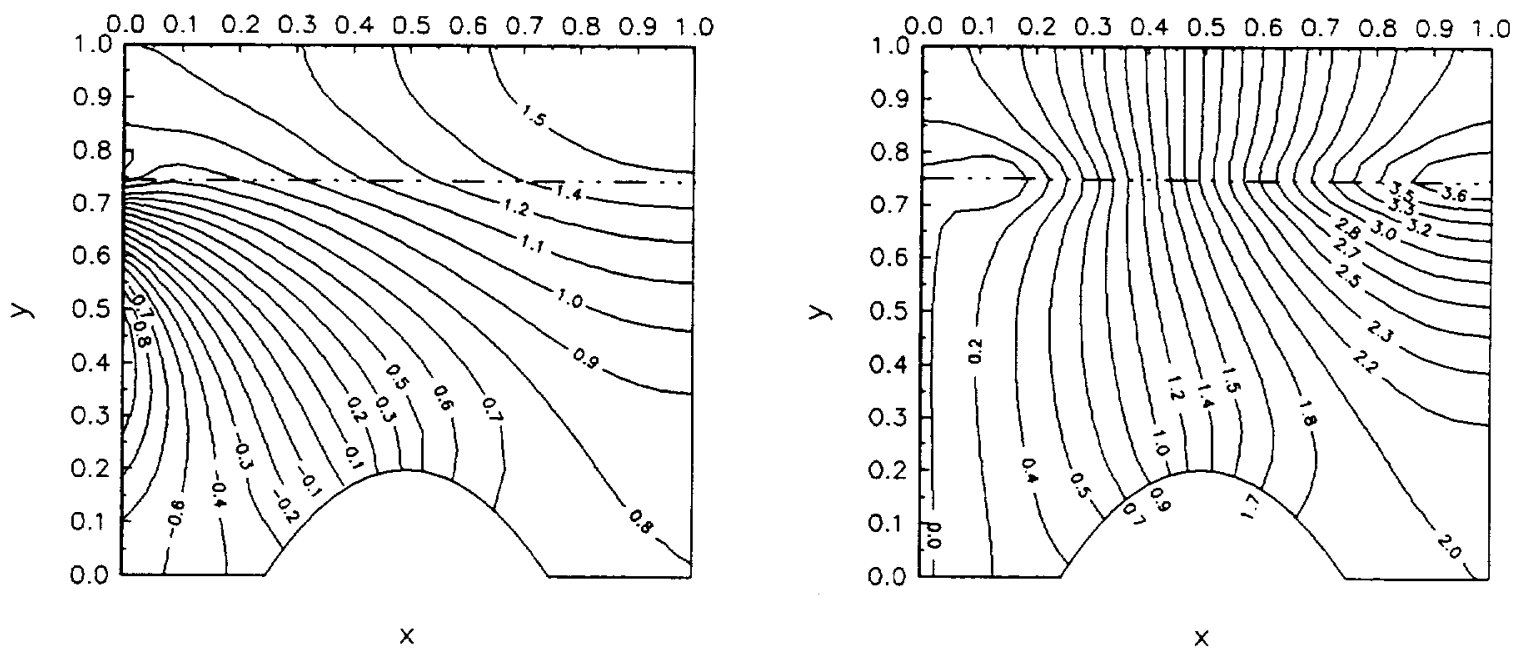

FIgURE 13. The contour plots for the temperature $T$ (left) and adjoint state $\Phi$ (right) $(\gamma=\delta=1)$. 

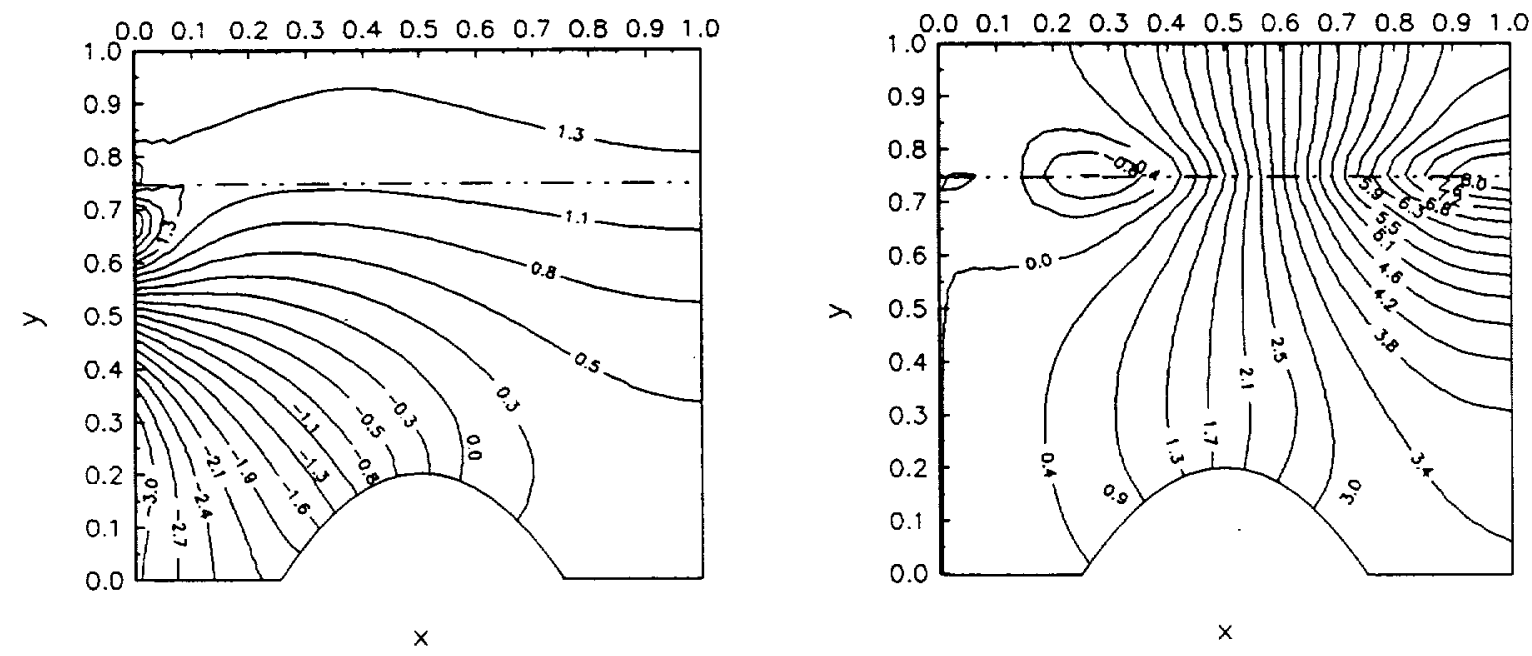

Figure 14. The contour plots for the temperature $T$ (left) and adjoint state $\Phi$ (right) $(\gamma=0.01, \delta=0.002)$.

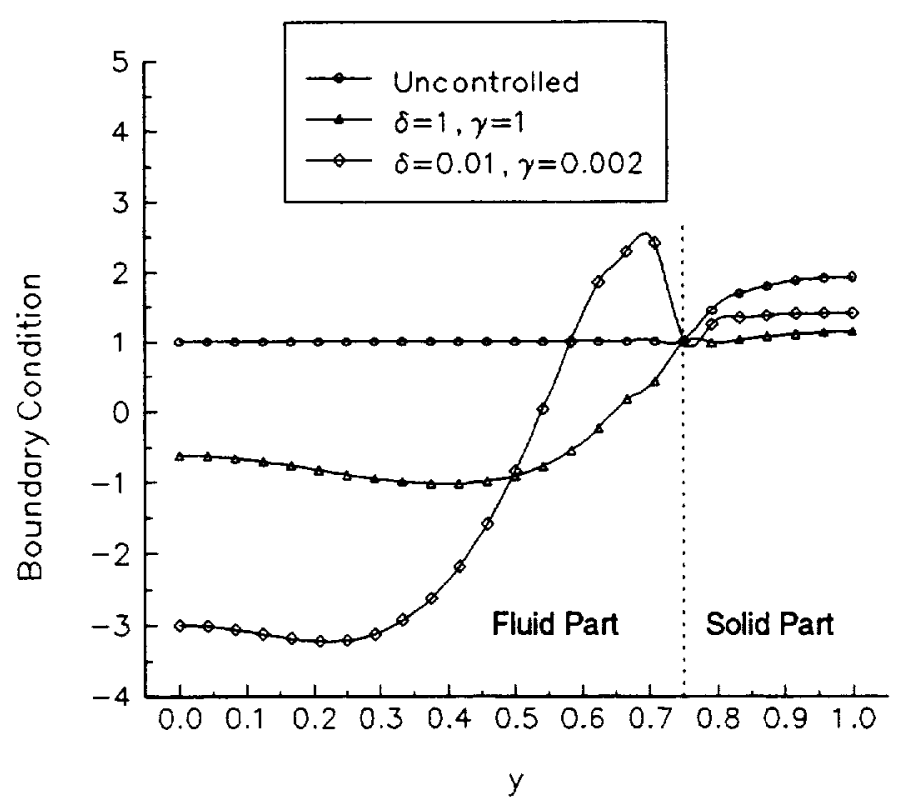

Figure 15. The optimal controls on $\Gamma_{c}$. 


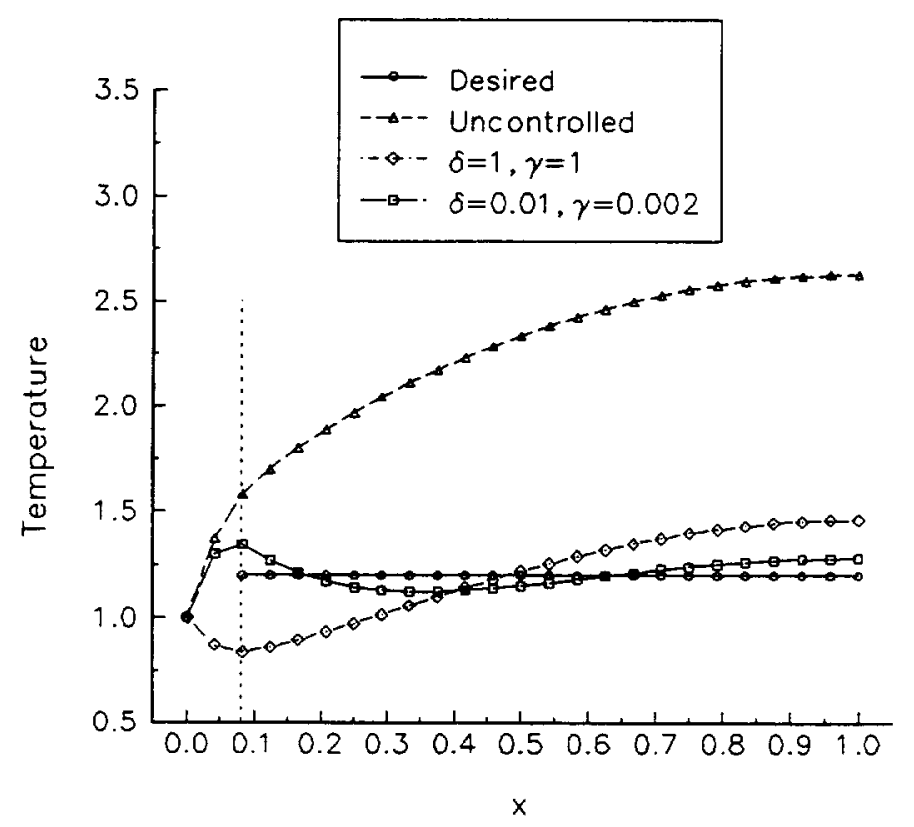

FIgURE 16. The temperature distributions on $\Gamma_{w}$.

\section{REFERENCES}

1. R. Adams, Sobolev Spaces, Academic, New York, 1975.

2. F. Abergel and R. Temam, On Some Control Problems in Fluid Mechanics, Theoret. Comput. Fluid Dynamics, 303-325, 1990.

3. V. Alekseev, V. Tikhomirov and S. Fomin, Optimal Control, Consultants Bureau, New York, 1987.

4. I. Babuška, The finite element method with Lagrangian multipliers. Numer. Math. 20, 179-192, 1973.

5. I. Babuška and A. Aziz, Survey lectures on the mathematical foundations of the finite element method. The Mathematical Foundations of the Finite Element Method with Applications to Partial Differential Equations (Ed. by A. Aziz), Academic, New York, 3-359, 1973.

6. F. Brezzi, J. Rappaz and P.-A. Raviart, Finite-dimensional approximation of nonlinear problems. Part I: branches of nonsingular solutions, Numer. Math. 36 1-25, 1980.

7. P. Ciarlet, The Finite Element Method for Elliptic Problems, North-Holland, Amsterdam, 1978.

8. P. Ciarlet, Introduction to Numerical Linear Algebra and Optimization, Cambridge, 1989

9. M. Crouziex and J. Rappaz, On Numerical Approximation in Bifurcation Theory, Masson, Paris, 1989.

10. P. Cuvelier, Optimal control of a system governed by the Navier-Stokes equations coupled with the heat equations, New Developments in Differential Equations(Ed. by W. Eckhaus), North-Holland, Amsterdam, 81-98, 1976. 
11. R. Dautray and J.-L. Lions, Mathematical Analysis and Numerical Methods for Science and Technology, Volume 1, Springer, Berlin, 1988.

12. R. Dautray and J.-L. Lions, Mathematical Analysis and Numerical Methods for Science and Technology, Volume 2, Springer, Berlin, 1988.

13. V. Girault and P.-A. Raviart, Finite element Methods for Navier-Stokes Equations, Springer, Berlin, 1986.

14. M. Gunzburger, L. Hou and T. Svobodny, Heating and Cooling Control of Temperature Distributions along Boundaries of Flow Domains, to appear.

15. M. Gunzburger, L. Hou and T. Svobodny, Treating inhomogeneous essential boundary conditions in finite element methods, SIAM J. Num. Anal., Vol 29, No.2, pp. 390-424, April 1992.

16. J.-L. Lions, Optimal Control of Systems governed by Partial Differential Equations, Springer, Berlin, 1971.

17. J. Serrin, Mathematical principles of classical fluid mechanics, Handbüch der Physik VIII/1 (Ed. by S flügge and C. Truesdell), Springer, Berlin, 125-263, 1959.

(Max D. Gunzburger) Department of Mathematics Virginia Polytechnic InstiTUTE aNd State UNIVERSity, Blacksburg, VA 24060, USA

E-mail address: gunzburger@vtcc1.cc.vt.edu

(Hyung C. Lee) Department of Mathematics Virginia Polytechnic Institute and STATE University, Blacksburg, VA 24060, USA

E-mail address: hlee@math.vt.edu 
Public reponting burden for this collection of information is estimated to average t hour per response, including the time for reviewing instructions, searching existing data sources, gathering and maintaining the data needed, and completing and reviewing the collection of information. Send comments regarding this burden estimate of any other aspect of this collection of information, including suggestions for reducing this burden, to Washington Headquarters Services. Directorate for Information Operations and Reports. 1215 Jefferson Davis Highway. Suite 1204. Arlington, VA 22202-4302, and to the Office of Management and Budget, Paperwork Reduction Projed (0704-0188), Washington, DC 20503.
1. AGENCY USE ONLY (Leave blank)
2. REPORT DATE
September 1993
3. REPORT TYPE AND DATES COVERED
Technical Memorandum

\section{TITLE AND SUBTITLE}

Analysis, Approximation, and Computation of a Coupled Solid/Fluid Temperature Control Problem

6. AUTHOR(S)

Max D. Gunzburger and Hyung C. Lee
5. FUNDING NUMBERS

WU-505-90-5K
B. PERFORMING ORGANIZATION REPORT NUMBER

E-8132

National Aeronautics and Space Administration

Lewis Research Center

Cleveland, Ohio 44135-3191

10. SPONSORING/MONITORING AGENCY REPORT NUMBER

National Aeronautics and Space Administration

Washington, D.C. 20546-0001

NASA TM-106351

ICOMP-93-30

\section{SUPPLEMENTARY NOTES}

Max D. Gunzburger, Institute for Computational Mechanics in Propulsion, NASA Lewis Research Center and Virginia Polytechnic Institute and State University, Blacksburg, Virginia 24061 (supported in part by U.S. Air Force Office of Scientific Research under grant number AFOSR-90-0179 and AFOSR-1-93-0061, by the Office of Naval Research

\begin{tabular}{|l|l|}
\hline 12a. DISTRIBUTIONAVAILABILTTY STATEMENT & 12b. DISTRIBUTION CODE \\
Unclassified - Unlimited & \\
Subject Category 64 & \\
\hline
\end{tabular}

\section{ABSTRACT (Maximum 200 words)}

An optimization problem is formulated motivated by the desire to remove temperature peaks, i.e., "hot spots", along the bounding surfaces of containers of fluid flows. The heat equation of the solid container is coupled to the energy equations for the fluid. Heat sources can be located in the solid body, the fluid, or both. Control is effected by adjustments to the temperature of the fluid at the inflow boundary. Both mathematical analyses and computational experiments are given.

\section{SUBJECT TERMS}

Optimal control; Navier-Stokes equation; Energy equation; Boundary control; Finite element methods; Gradients method

\begin{tabular}{|c|c|c|}
\hline $\begin{array}{c}\text { 17. SECURTY CLASSIFICATION } \\
\text { OF REPORT } \\
\text { Unclassified }\end{array}$ & $\begin{array}{c}\text { 18. SECURTY CLASSIFICATION } \\
\text { OF THIS PAGE } \\
\text { Unclassified }\end{array}$ & $\begin{array}{c}\text { 19. SECURITY CLASSIFICATION } \\
\text { OF ABSTRACT } \\
\text { Unclassified }\end{array}$ \\
\hline
\end{tabular}

15. NUMBER OF PAGES 34 16. PRICE CODE $\mathrm{AO3}$ 20. LIMITATION OF ABSTRACT 


\section{Supplementary Notes}

under grant number N00014-91-J-1493, and by NASA Cooperative Agreement NCC3-233) and Hyung C. Lee, Virginia Polytechnic Institute and State University, Blacksburg, Virginia 24061, (work funded under NASA Cooperative Agreement NCC3-233). ICOMP Program Director, Louis A. Povinelli, (216) 433-5818. 